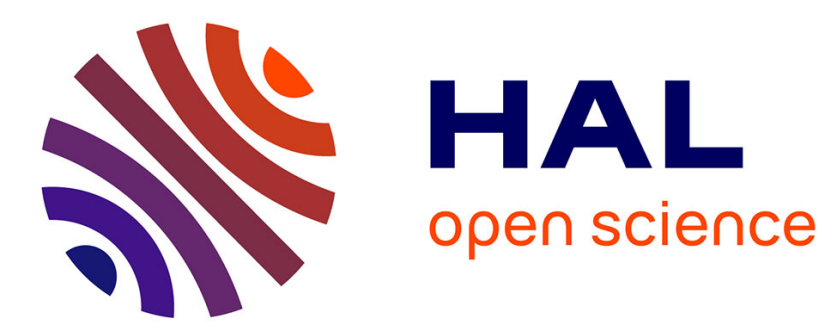

\title{
Eulerian quadrature-based moment models for dilute polydisperse evaporating sprays
}

Damien Kah, Frédérique Laurent, Lucie Fréret, Stephane de Chaisemartin, Rodney Fox, Julien Reveillon, Marc Massot

\section{- To cite this version:}

Damien Kah, Frédérique Laurent, Lucie Fréret, Stephane de Chaisemartin, Rodney Fox, et al.. Eulerian quadrature-based moment models for dilute polydisperse evaporating sprays. Flow, Turbulence and Combustion, 2010, 85, pp.649-676. 10.1007/s10494-010-9286-z . hal-00449866

\section{HAL Id: hal-00449866 https://hal.science/hal-00449866}

Submitted on 23 Jan 2010

HAL is a multi-disciplinary open access archive for the deposit and dissemination of scientific research documents, whether they are published or not. The documents may come from teaching and research institutions in France or abroad, or from public or private research centers.
L'archive ouverte pluridisciplinaire HAL, est destinée au dépôt et à la diffusion de documents scientifiques de niveau recherche, publiés ou non, émanant des établissements d'enseignement et de recherche français ou étrangers, des laboratoires publics ou privés. 


\title{
Submitted for the Special Issue dedicated to S. B. Pope \\ Eulerian quadrature-based moment models for dilute polydisperse evaporating sprays
}

\author{
Damien Kah · Frédérique Laurent · Lucie Fréret . \\ Stéphane de Chaisemartin · Rodney O. Fox · Julien \\ Reveillon · Marc Massot
}

Received: date / Accepted: date

\begin{abstract}
Dilute liquid sprays can be modeled at the mesoscale using a kinetic equation, namely the Williams-Boltzmann equation, containing terms for spatial transport, evaporation and fluid drag. The most common method for simulating the Williams-Boltzmann equation uses Lagrangian particle tracking wherein a finite ensemble of numerical "parcels" provides a statistical estimate of the joint surface area, velocity number density function (NDF). An alternative approach is to discretize the NDF into droplet size intervals, called sections, and to neglect velocity fluctuations conditioned on droplet size, resulting in an Eulerian multi-fluid model. In comparison to Lagrangian particle tracking, multi-fluid models contain no statistical error (due to the finite number of parcels) but they cannot reproduce the particle trajectory crossings observed in Lagrangian simulations of non-collisional kinetic equations. Here, in order to overcome this limitation, a quadrature-based moment method is used to describe the velocity moments. When coupled with the sectional description of droplet sizes, the resulting Eulerian multi-fluid, multi-velocity model is shown to capture accurately both particle trajectory crossings and the size-dependent dynamics of evaporation and fluid drag. Model validation is carried out using direct comparisons between the Lagrangian and Eulerian models for an unsteady free-jet configuration with mono- and polydisperse droplets with and without evaporation. Comparisons between the Eulerian and Lagrangian instantaneous number density and gas-phase fuel mass fraction fields show excellent agreement, suggesting that the multi-fluid, multi-velocity model is well suited for describing spray combustion.
\end{abstract}

Keywords Dilute polydisperse spray · Williams-Boltzmann equation · Quadrature-based moment methods $\cdot$ Eulerian multi-fluid model $\cdot$ Eulerian multi-velocity model

R. O. Fox

Dept. of Chemical and Biological Engineering, Iowa State University, USA. E-mail: rofox@iastate.edu

D. Kah

Institut Français du Pétrole, Rueil-Malmaison, and Laboratoire EM2C-UPR CNRS 288, Ecole Centrale Paris, Châtenay-Malabry, France

F. Laurent, L. Fréret, M. Massot

Laboratoire EM2C-UPR CNRS 288, Ecole Centrale Paris, Châtenay-Malabry, France

S. de Chaisemartin

Institut Français du Pétrole, Rueil-Malmaison, France

J. Reveillon

CORIA - UMR CNRS 6614, Université de Rouen, Saint Etienne du Rouvray, France 


\section{Introduction}

Many industrial devices involve turbulent combustion of a liquid fuel. Indeed, the transportation sector, rocket, aircraft and car engines are almost exclusively based on storage and injection of a liquid phase, which is sprayed into a chamber where turbulent combustion takes place. Thus, it is of primary importance to understand and control the physical process as a whole, from the injection into the chamber up to the combustion phenomena. Numerical simulation is now a standard tool to optimize turbulent combustion processes in such devices. If the modeling of purely gas-phase configurations is relatively well understood with a wide range of suggested closures such as the transported probability density function methods pioneered by S. B. Pope [22], this is not the case for two-phase flows where detailed information is needed about the physics of the triple interactions of spray dynamics, fluid turbulence and combustion.

In general, two approaches for treating liquid sprays, corresponding to two levels of description, can be identified. The first one, associated with a full direct numerical simulation (DNS) of the process, provides a model for the dynamics of the interface between the gas and liquid phases, as well as for the details of the exchange of heat and mass between the two phases. The second one, based on a more global point of view, uses kinetic theory to describe the droplets as a cloud of point particles, the geometries of which are presumed spherical, and for which the exchange of mass, momentum and heat are described globally. The latter is the only description for which numerical simulations at the scale of a combustion chamber can be conducted. Thus, this "mesoscopic" point of view will be adopted in the present study.

In the kinetic theory framework, there exists considerable interest in the development of numerical methods for simulating sprays using the Williams-Boltzmann transport equation [26]. The principal physical processes that must be accounted for are (1) transport in physical space, (2) evaporation, (3) size-dependent acceleration of droplets due to drag, and (4) breakup, rebound and coalescence leading to polydispersity. The major challenge in numerical simulations is to account for the strong coupling between these processes. In the context of one-way coupling, the Lagrangian Monte-Carlo approach (also known as direct simulation Monte-Carlo (DSMC) [2]) is generally considered to be more accurate than Eulerian methods for solving the Williams-Boltzmann equation. However, its computational cost is high, especially in unsteady configurations. Moreover, in applications with two-way coupling, Lagrangian methods are difficult to couple accurately with Eulerian descriptions of the gas phase. Thus, there is considerable impetus to develop Eulerian methods, keeping in mind that such models still need validation.

Currently there exists two significant shortcomings in Eulerian models. First, they fail to describe polydispersity. However, in many industrial configurations, evaporating droplets of different sizes follow different pathways, depositing their fuel mass fraction at different locations. One way to overcome this shortcoming is to use multi-fluid models $[16,18,4,19]$. Second, Eulerian models are derived from the Williams-Boltzmann equation through an near-equilibrium assumption (called the hydrodynamic limit for the normal solution of the Boltzmann equation in kinetic theory $[13,1])$, leading to closure at the level of second-order velocity moment equations conditioned on droplet size. For dilute sprays (e.g. liquid volume fractions of less than one percent), droplet-droplet collisions are negligible and, hence, the important processes leading to an equilibrium velocity distribution in the Boltzmann equation are absence. Since it is essentially monokinetic (i.e., near equilibrium), the hydrodynamic model is unable to capture the multi-modal droplet velocity distributions arising in dilute sprays during droplet crossings. Even if the multi-fluid model can capture droplet 
crossing for droplets of different sizes, the near-equilibrium assumption is too limiting and leads to the creation of singularities (i.e. ' $\delta$-shocks') that have been studied analytically in [18], with a physical interpretation in [5,4]. Recently, the development of quadrature-based moment methods in velocity phase space $[8,9]$ has provided a closure for non-equilibrium velocity distributions for monodisperse particles, providing a description of droplet crossing at finite Stokes numbers. In principle, by adding the collision terms to the kinetic equations [11], quadrature-based moment methods can treat liquid sprays with any liquid volume fraction, and thus have the potential to overcome all of the known shortcomings of Eulerian models for polydisperse two-phase flows.

The framework of the present study is DNS of the gas phase with one-way coupling to the kinetic equation describing the liquid phase. However, in the context of large-eddy simulations, Eulerian models will encounter the same issues described above from both a modeling and computational point of view. Furthermore, in this study, we evaluate the numerical methods in a 2-D framework. Nevertheless, the models can be easily extended to 3-D configurations [4].

The scope of the present contribution is two fold. First, an evaluation of the multifluid model in a free-jet configuration is carried out by a detailed comparison between the MUSES3D ${ }^{1}$ code [4] and the Euler-Lagrange ASPHODELE solver [25,24]. After demonstrating the accuracy of the multi-fluid model for capturing the dynamics of droplets of various sizes, we investigate its ability to properly evaluate the gas-phase fuel mass fraction field issuing from evaporation. For droplets with moderate Stokes number, the proposed numerical scheme, which is second order in time and space, treats the potential singularities naturally occurring in the model equations and attains a very satisfactory level of accuracy with very limited numerical diffusion. Properly capturing the topology of the fuel mass fraction resulting from evaporation is the primary goal of a spray model for combustion applications and we demonstrate the necessity of describing accurately the polydispersity in order to reach this goal.

Second, a new Eulerian model, with dedicated numerical schemes, able to deal with polydispersity as well as non-equilibrium velocity distributions for evaporating sprays based on the quadrature method of moments in velocity phase space conditioned on droplet size is developed. Two key issues are addressed (beyond the techniques introduced in [8,9]): (1) moment space must to be preserved, that is the numerical method must guarantee that the moment vectors throughout the computation always remain moments of a velocity distribution when transport is coupled to drag and evaporation; and (2) the higher-order model must naturally degenerate to the multi-fluid model at the boundaries of moment space, that is when the velocity distribution function becomes monokinetic up to machine precision. Using Lagrangian/Eulerian comparisons, we illustrate the ability of the newly developed model and numerical methods to satisfy these properties. Comparisons between the multifluid model and the higher-order multi-fluid, multi-velocity model in a free-jet configuration with two polydisperse spray injections are presented. We emphasize the necessity to capture droplet trajectory crossing in such a case and again demonstrate the good performance of the proposed model.

The organization of the paper is as follows. After briefly recalling the fundamentals of both the Lagrangian discrete particle simulations (DPS) and the multi-fluid model (as well as the associated numerical methods) in Sects. 2 and 3, we focus our attention in Sect. 4 on the free-jet configuration with polydisperse spray injection and delineate the accuracy and efficiency of the multi-fluid model and numerical methods, as well as its limitations.

\footnotetext{
1 Multi-fluid Spray Eulerian Solver developed at EM2C by L. Fréret and S. de Chaisemartin.
} 
In Sect. 5, the multi-fluid, multi-velocity model is introduced. We investigate the details of the quadrature method (which is a key issue) and the numerical method needed to preserve the moment space. Section 6 is devoted to the numerical investigation of a single free jet with droplets over a large range of Stokes numbers leading to droplet crossing. The ability to properly capture the behavior on the boundaries of moment space is presented, as well as the necessity to rely on a multi-velocity model for a two-jet configuration. The principal achievements of the present contribution are summarized in the Conclusions.

\section{Statistical description at the mesoscopic scale and Lagrangian discretization}

At the mesoscopic scale, liquid sprays are described as a cloud of point particles for which the exchange of mass, momentum and thermal energy are described globally, using eventually correlations, and the details of the interface behavior, angular momentum of droplets, etc., are not predicted. In the following, even if heating can easily be included in the models, we will restrict the framework of the study to liquid sprays undergoing evaporation and drag. We also make the assumption that these phenomena only depend on the local gasphase properties as well as on the state of each droplet. In addition, we assume that all the scales of the gas phase are resolved in the context of DNS. Moreover, we restrict our attention to dilute sprays where coalescence, breakup and collisions in general can be neglected. It should be noted that the models have been extended to more dense sprays, where droplets coalescence [16] or rebounds [12] can take place. We adopt a statistical (kinetic) description of the Boltzmann type and the spray can be described by its joint surface area $(S)$, velocity (u) number density function (NDF) $f(t, \mathbf{x}, S, \mathbf{u})$, which satisfies the following Williams-Boltzmann equation [26]:

$$
\partial_{t} f+\partial_{\mathbf{x}} \cdot(\mathbf{u} f)+\partial_{S}(K f)+\partial_{\mathbf{u}} \cdot(\mathbf{F} f)=0,
$$

where, for the sake of simplicity, $K(t, \mathbf{x})$ is the constant of a $d^{2}$ law and $\mathbf{F}=\left(\mathbf{U}_{\mathrm{g}}(t, \mathbf{x})-\right.$ $\mathbf{u}) / \tau_{\mathrm{p}}(S)$ is the Stokes drag force per unit mass, $\mathbf{U}_{\mathrm{g}}$ being the gas velocity and $\tau_{\mathrm{p}}(S)=$ $\rho_{\mathrm{l}} S /\left(\rho_{\mathrm{g}} 18 \pi v\right)$ is the droplet dynamical time, where $\rho_{\mathrm{l}}$ and $\rho_{\mathrm{g}}$ are the liquid and gas densities, respectively, and $v$ is the kinematic viscosity of the gas.

For the sake of simplicity, the liquid and gas densities as well as the gas viscosity are assumed constant here. This is partially justified by the fact that we will only consider configurations with a constant composition and temperature of the gas, but this is not a restriction of the model. Rather, it allows us to use a simple non-dimensional formulation, using a reference droplet surface $S_{0}$, a reference length $L_{0}$ for the space location, a reference velocity $U_{0}$ for the gas and droplet velocities, and the associated time scale $t_{0}=L_{0} / U_{0}$. The same notation is used for the dimensionless variables in such a way that the transport equation is also defined by Eq. (1), but with $K$ the non-dimensional evaporation rate (independent of $t$ and $\mathbf{x})$ and $\mathbf{F}=\left(\mathbf{U}_{\mathrm{g}}(t, \mathbf{x})-\mathbf{u}\right) /(\mathrm{St} S)$ the non-dimensional drag force, where St $=\tau_{\mathrm{p}}\left(S_{0}\right) / t_{0}$ is the Stokes number.

In this context, the Williams-Boltzmann equation can be discretized through a particle discretization (PD), where the NDF is represented by a sum of Dirac delta functions: $f(t, \mathbf{x}, \mathbf{u}, S)=\sum_{p} w_{p} \delta\left(\mathbf{x}-\mathbf{x}_{p}(t)\right) \delta\left(\mathbf{u}-\mathbf{u}_{p}(t)\right) \delta\left(S-S_{p}(t)\right)$, where $w_{p}$ is a constant weight of the $p^{\text {th }}$ numerical particle and $\mathbf{x}_{p}, \mathbf{u}_{p}, S_{p}$ are its position, velocity and surface area, respectively. These characteristics of numerical particles evolve through standard differential equations:

$$
\mathrm{d}_{t} \mathbf{x}_{p}=\mathbf{u}_{p}, \quad \mathrm{~d}_{t} \mathbf{u}_{p}=\mathbf{F}, \quad \mathrm{d}_{t} S_{p}=K .
$$


The PD method provides, if enough numerical particles are used, an ensemble average of the droplet number density and other relevant statistical quantities, which are Eulerian fields. Under the particular set of assumptions we have chosen, the PD method is equivalent to an ensemble of discrete particle simulations where each individual numerical particle represents one droplet and the weights are equal to one [25]. The number density of particles for DPS is then evaluated with respect to a given equivalence ratio for evaporation and combustion purposes, and corresponds to one realization of an ensemble average governed by the Williams-Boltzmann equation.

\section{Eulerian multi-fluid model}

As an alternative to Lagrangian methods, multi-fluid models have been developed, which take into account the polydispersity of the spray in a Eulerian formalism, while keeping a rigorous link to the kinetic model.

\subsection{Model equations}

The formalism and the associated assumptions needed to derive the multi-fluid model were originally introduced in [16], extending the ideas of [14]. We recall briefly the main features.

[H1] We presume the form of the $\operatorname{NDF} f(t, \mathbf{x}, S, \mathbf{u})=n(t, \mathbf{x}, S) \delta(\mathbf{u}-\overline{\mathbf{u}}(t, \mathbf{x}, S))$ through a single-node quadrature method of moments in velocity phase space conditioned on size, where $\overline{\mathbf{u}}(t, \mathbf{x}, S)$ is the average velocity conditioned on droplet size ${ }^{2}$.

[H2] The droplet size phase space is divided into intervals $\left[S_{k-1}, S_{k}\right.$ [, called sections. In one section, $\overline{\mathbf{u}}^{(k)}$ does not depend on droplet size and the form of $n^{(k)}(t, \mathbf{x}, S)=m^{(k)}(t, \mathbf{x}) \kappa^{(k)}(S)$ as a function of $S$ is assumed independent of $(t, \mathbf{x})$. The variable used is $m^{(k)}=\int_{S_{k-1}}^{S_{k}} \rho_{1} S^{3 / 2} n^{(k)} \mathrm{d} S$, the non-dimensional mass density in section $k$ relative to the typical mass density, $m_{0}=$ $\rho_{\mathrm{l}_{0}} S_{0}^{3 / 2} n_{0} /(6 \sqrt{\pi})$.

The set of droplets in one section can be seen as a 'fluid' for which conservation equations are written, thus yielding exchanges of mass and momentum between the coupled fluids. Droplets in different sections can then have different dynamics with an a priori control of the required precision in size phase space. Let us note that such an approach only focuses on one moment of the distribution in the size variable within each section, and the mass moment is chosen because of its relevance in evaporation and combustion processes. Higher-order approximations can also be used (see [20] and references therein).

The conservation equations for the $k^{\text {th }}$ section read:

$$
\begin{aligned}
\partial_{t} m^{(k)}+\partial_{\mathbf{x}} \cdot\left(m^{(k)} \overline{\mathbf{u}}^{(k)}\right)= & \left(E_{1}^{(k)}+E_{2}^{(k)}\right) m^{(k)}-E_{1}^{(k+1)} m^{(k+1)} \\
\partial_{t}\left(m^{(k)} \overline{\mathbf{u}}^{(k)}\right)+\partial_{\mathbf{x}} \cdot\left(m^{(k)} \overline{\mathbf{u}}^{(k)} \otimes \overline{\mathbf{u}}^{(k)}\right)= & \left(E_{1}^{(k)}+E_{2}^{(k)}\right) m^{(k)} \overline{\mathbf{u}}^{(k)} \\
& -E_{1}^{(k+1)} m^{(k+1)} \overline{\mathbf{u}}^{(k+1)}+m^{(k)} \overline{\mathbf{F}}^{(k)}
\end{aligned}
$$

where $E_{1}^{(k)}$ and $E_{2}^{(k)}$ are the evaporation coefficients and $\overline{\mathbf{F}}^{(k)}=\left(\mathbf{U}_{\mathrm{g}}(t, \mathbf{x})-\mathbf{u}\right) /\left(\mathrm{St} S_{\text {mean }}^{(k)}\right)$ is the average drag force, a function of the mean surface area of the section $S_{\text {mean }}^{(k)}$. For a

\footnotetext{
2 This corresponds to a generalized Maxwell-Boltzmann distribution at zero temperature and remains an "equilibrium" velocity distribution even if there is no collision operator in the model.
} 
choice of the shape of the distribution with $\kappa^{(k)}(S)$ constant in each section, the evaporation coefficients can be written:

$$
E_{1}^{(k)}=\frac{5 S_{k-1}^{3 / 2}}{2\left(S_{k}^{5 / 2}-S_{k-1}^{5 / 2}\right)} K, \quad E_{2}^{(k)}=\frac{5\left(S_{k}^{3 / 2}-S_{k-1}^{3 / 2}\right)}{2\left(S_{k}^{5 / 2}-S_{k-1}^{5 / 2}\right)} K, \quad S_{\text {mean }}^{(k)}=\frac{3\left(S_{k}^{5 / 2}-S_{k-1}^{5 / 2}\right)}{5\left(S_{k}^{3 / 2}-S_{k-1}^{3 / 2}\right)} .
$$

The $E_{1}^{(k)}$ and $E_{2}^{(k)}$ terms represent the exchange between successive sections and exchange with the gas phase through evaporation, respectively. These conservation equations have the same mathematical structure as the pressure-less gas dynamics equation. Thus, they potentially lead to singular behavior and require well-suited numerical methods $[19,5]$.

\subsection{Numerical methods}

Because of the transport in physical space and the transport in phase space due to evaporation and drag have different structures, we use a Strang splitting algorithm [5, 18]. We first solve for $\Delta t / 2$ the transport in phase space, then for $\Delta t$ the transport in physical space, and then for $\Delta t / 2$ the transport in phase space. The interest in Strang splitting is two fold. First, this approach has the great advantage of preserving the properties of the schemes we use for the different contributions, such as for example a maximum principle or positivity. If we assume that the involved phenomena evolve at roughly the same time scales, the Strang splitting algorithm guarantees second-order accuracy in time provided that each of the elementary schemes has second-order time accuracy. Furthermore, from a computational point of view, this is optimal and yields high parallelization capabilities.

The transport in physical space obeys a system of weakly hyperbolic conservation laws and relies on kinetic finite volume schemes as introduced in [3] in order to solve the pressureless gas dynamics equation. Through assumption [H1], it defines a kinetic description that is equivalent to the moment system of equations for smooth solutions and allows to properly define the fluxes for transport of the moments in one space dimension. The resulting scheme is second-order accurate in space and time. For a 2-D space, we further use a dimensional Strang splitting of the 1-D scheme previously described in [5]. The corresponding scheme offers the ability to treat the $\delta$-shocks and vacuum states, and preserves the positivity of the mass density as well as the moment space.

For the transport in phase space through evaporation and drag, the model equations reduce to systems of ODE's, which can be stiff, for each point of the domain. The system is solved using an implicit Runge-Kutta Radau IIA method of order 5 with adaptive time steps.

\section{Results with Eulerian multi-fluid model}

The aim of this section is first to validate the Eulerian multi-fluid model on an unsteady flow configuration. We then show the importance of the description of the polydispersity, and also highlight some of the limitations of the multi-fluid model for describing dilute flows.

\subsection{Free-jet configuration}

In order to assess the Eulerian methods we focus on a 2-D free jet. A polydisperse spray is injected in the jet core with either a lognormal size NDF (Figure 1-right), whose mean diam- 
eter $d_{0}$ corresponds to the reference surface $S_{0}$, or a constant size distribution on $\left[0, S_{0}\right]$ in the surface variable (linear in radius), corresponding to the beginning of a typical experimental distribution [17]. The simulations are conducted with an academic solver, coupling the ASPHODELE solver [23] with the multi-fluid solver MUSES3D [4, 19], using the models presented in this work. The ASPHODELE solver couples a Eulerian description of the gas phase with a Lagrangian description of the spray. One of the key features of this simulation tool is to allow, in the framework of one-way coupling, the simultaneous computation of the gas phase as well as both Lagrangian and Eulerian spray descriptions within the same code.
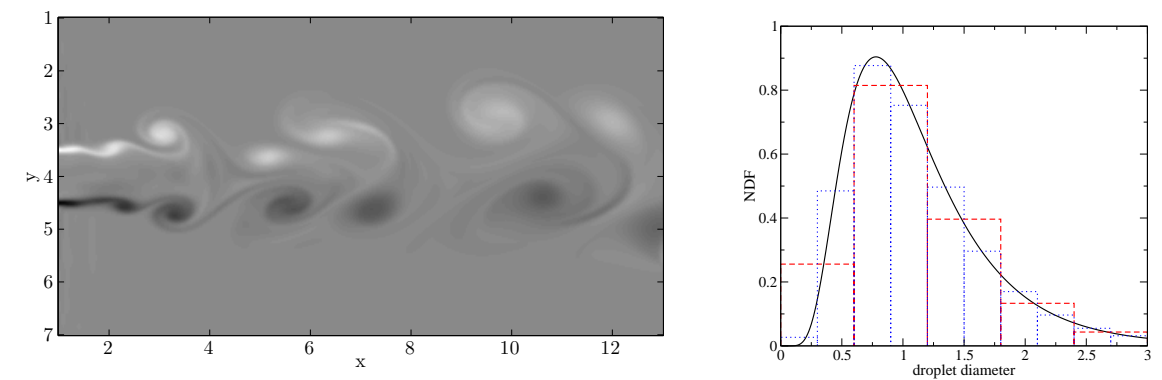

Fig. 1 Free-jet configuration at time $t=20$. (Left) Gas vorticity on a $400 \times 200$ grid. (Right) Polydisperse lognormal distribution discretized with 5 and 10 sections.

As far as the gas phase is concerned, we use a 2-D Cartesian low Mach number compressible solver. The gas jet is computed on a $400 \times 200$ uniformly spaced grid. To destabilize the jet, we inject turbulence using the Klein method with $10 \%$ fluctuations [15]. The Reynolds number based on $U_{0}, v_{0}$ and $L_{0}$ is 1,000 , where $U_{0}$ is the injection velocity and $L_{0}$ is the jet width. We will eventually provide dimensional quantities for illustration purposes. These will be based on a velocity of $U_{0}=1 \mathrm{~m} / \mathrm{s}$ and $L_{0}=1.5 \times 10^{-2} \mathrm{~m}$, as well as a typical value of $v_{0}=1.6 \times 10^{-5} \mathrm{~m}^{2} / \mathrm{s}$. Finally we have $d_{0}=L_{0} / 300$, where $d_{0}$ is the diameter corresponding to the typical droplet surface $S_{0}$, and $\rho_{\mathrm{l}} / \rho_{\mathrm{g}}=565$. The gas vorticity is presented in Figure 1-left. Since we aim to validate the Eulerian models through comparisons to a Lagrangian simulation, and to show the importance of the description of the polydispersity, we restrict ourselves to one-way coupling.

\subsection{Lagrangian versus multi-fluid model for free-jet configuration}

In this first case, the lognormal distribution (Figure 1-right) is used for the injected spray. We take as a reference solution for the liquid phase a Lagrangian DPS with particle numbers in the computational domain ranging from 10,000 to 70,000 depending on the case. The number of droplets for each case is determined by stoichiometry. We provide comparisons between the Lagrangian reference and the Eulerian multi-fluid computations by plotting the Lagrangian particle positions versus the Eulerian number density. Thanks to the multi-fluid description, we perform the comparisons for different ranges of droplet sizes and thus for different Stokes numbers, for evaporating and non-evaporating cases. 


\subsubsection{Free-jet non-evaporating test case}

For the non-evaporating case we use five sections for the multi-fluid simulation (see Figure 1-right). We have 70,000 Lagrangian particles in the computational domain at the time considered. We present first a comparison for low-inertia droplets and find a very good agreement for the droplets with a Stokes range from 0.011 to 0.12 , corresponding to diameters between $9 \mu \mathrm{m}$ and $30 \mu \mathrm{m}$, as shown in Figure 2-left. The multi-fluid model is thus shown to simulate the dynamics of a polydisperse spray for relatively small Stokes numbers. Droplet dynamics are close to the gas dynamics for this range of sizes, and therefore the model remains in its domain of validity (see Sect. 3). For higher Stokes numbers the droplets are ejected from the vortices and crossing trajectories are likely to occur, breaking the monokinetic multi-fluid assumption described in Sect. 3. Nevertheless, the dynamics are still very well reproduced for high-inertia droplets. The results are plotted in Figure 2-right for Stokes numbers from 0.48 to 1.1 , corresponding to diameters from $60 \mu \mathrm{m}$ to $90 \mu \mathrm{m}$. One can notice that the number density is concentrated in a few cells in this case and that the numerical method does not encounter any problems to capture the distribution, illustrating its robustness.
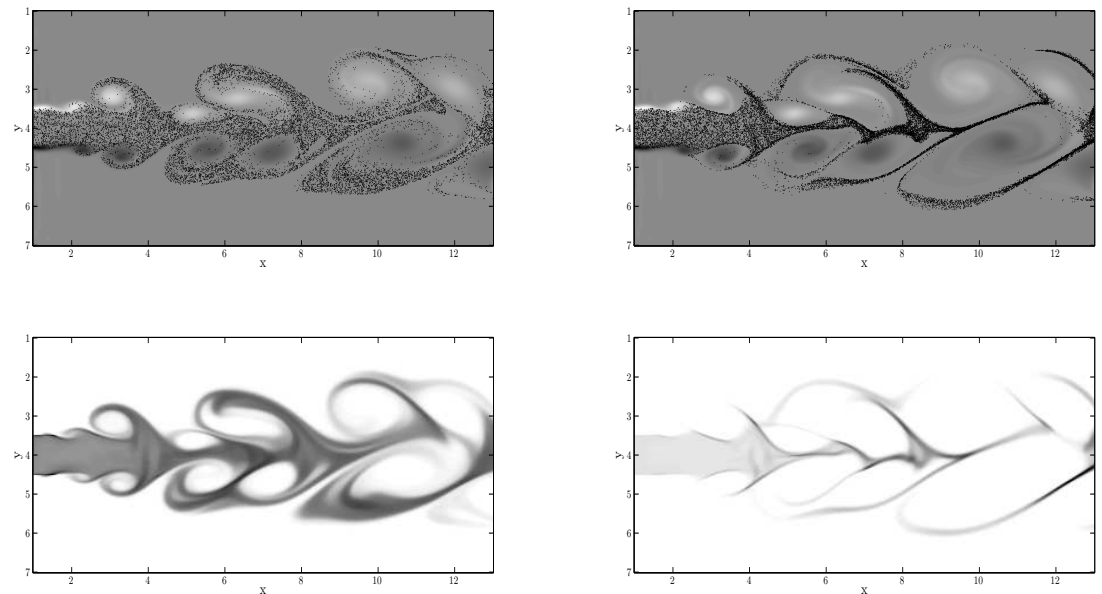

Fig. 2 Non-evaporating polydisperse spray at time $t=20$. (Left) Low-inertia droplets with Stokes 0.011 to 0.12 , corresponding to diameters from 9 to $30 \mu \mathrm{m}$. (Right) High-inertia droplets with Stokes 0.48 to 1.1, corresponding to diameters from 60 to $90 \mu \mathrm{m}$. (Top) Lagrangian particle positions with 40,000 particles over gas vorticity. (Bottom) Eulerian number density on a $400 \times 200 \times 5$ grid.

\subsubsection{Free-jet evaporating test case}

The free-jet case is assessed here with an evaporating spray. For the $d^{2}$ law, we take a constant mass-transfer number $\mathrm{Bm}=0.1$. The corresponding non-dimensional evaporation coefficient is $K=0.07$. The results are presented in the same manner as for the non-evaporating case. In order to describe accurately the evaporation process, we take ten sections for the 
multi-fluid simulation, whereas 30,000 Lagrangian particles are present in the domain at the time considered. As in the non-evaporating case, we find a very good agreement between the Eulerian and Lagrangian descriptions. For low-inertia droplets, the comparison is shown in Figure 3-left, with Stokes numbers from 0.011 to 0.12 , corresponding to diameters $d_{0}=9 \mu \mathrm{m}$ to $d_{0}=30 \mu \mathrm{m}$. For high-inertia droplets, the comparison is shown in Figure 3right, with Stokes number from 0.48 to 1.1 , corresponding to diameters from $60 \mu \mathrm{m}$ to $90 \mu \mathrm{m}$.
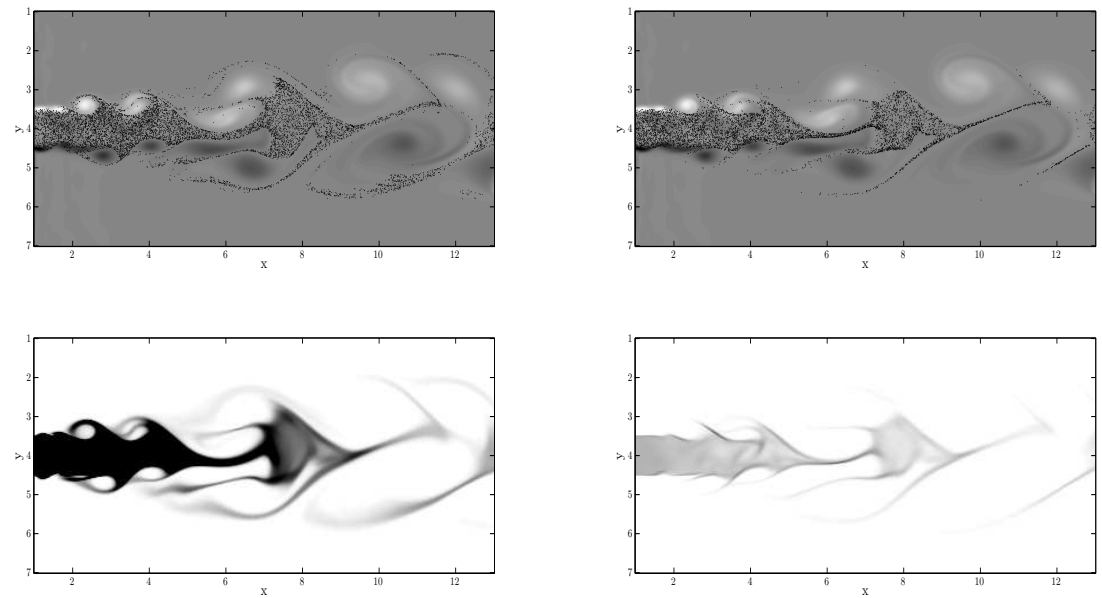

Fig. 3 Evaporating polydisperse spray at time $t=20$. (Left) Low-inertia droplets with Stokes 0.011 to 0.12 , corresponding to diameters from 9 to $30 \mu \mathrm{m}$. (Right) High-inertia droplets with Stokes 0.48 to 1.1 , corresponding to diameters from 60 to $90 \mu \mathrm{m}$. (Top) Lagrangian particle positions with 40,000 particles over gas vorticity. (Bottom) Eulerian number density on a $400 \times 200 \times 5$ grid.

The polydisperse evaporating free-jet case shows the ability of the multi-fluid method to treat more complex flows, closer to realistic configurations. Using these comparisons, we demonstrate that the multi-fluid model captures size-conditioned dynamics that carry droplets of different sizes to different locations. It is then essential to evaluate the ability of the Eulerian model to capture the evaporation process as a whole.

\subsubsection{Gas-phase fuel mass fraction}

Our interest being in combustion applications, a key issue of evaporating spray modeling is prediction of the gas-phase fuel mass fraction. We thus present comparisons between the gas-phase fuel mass fraction obtained from the Lagrangian and Eulerian descriptions of the spray. These results are found with the same coupled codes used in the previous section, the spray being described on the one hand by the Lagrangian method and on the other hand by the multi-fluid model. These simulations are again done using one-way coupling. As a consequence, the evaporated fuel is not added as a mass source term in the gas-phase equations, but is stored in two passive scalars, one for each description of the spray, that are transported by the flow. The Lagrangian gas-phase fuel mass fraction is obtained through 
a projection of the droplet evaporation over the neighbor cells of the computational mesh. These two fields are plotted in Figure 4. One can see the very good agreement of both descriptions for spray evaporation. This comparison underlines the efficiency of the multifluid model in describing polydisperse evaporating sprays. Furthermore, as can be seen in Figure 4, the Eulerian description provides a smoother field than the Lagrangian one. This illustrates the difficulties that arise when coupling the Lagrangian description of the liquid to the Eulerian description of gas, and underlines the advantage of the Eulerian description of the spray for the liquid-gas coupling. These results represent a first step towards combustion computations with full two-way coupling.
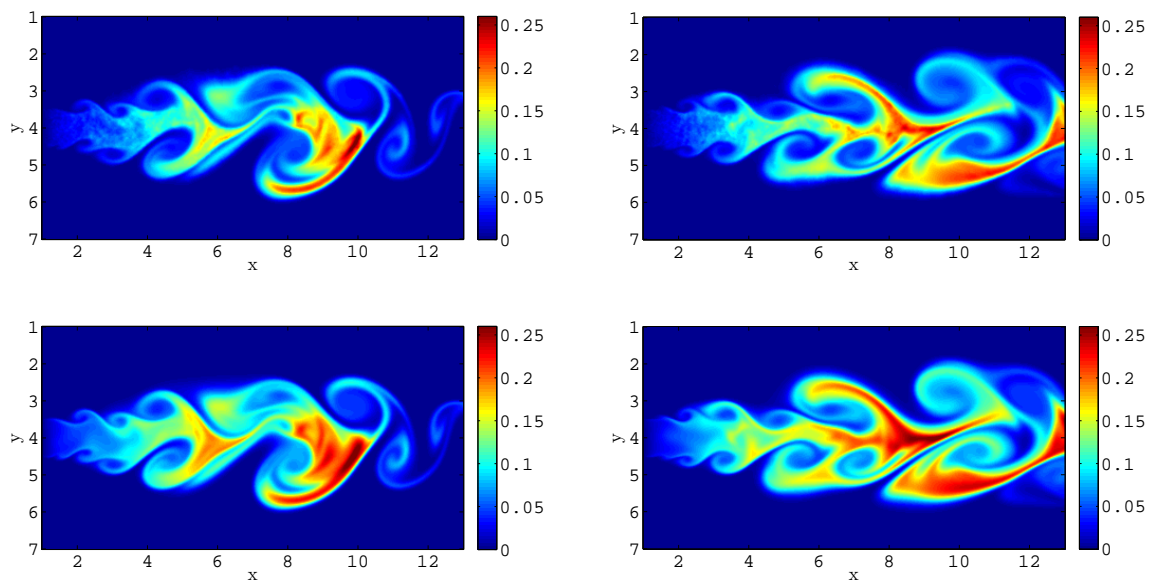

Fig. 4 Comparison of the gas-phase fuel mass fraction at times $t=15$ (left) and $t=20$ (right). (Top) Lagrangian method with 30,000 droplets. (Bottom) Eulerian multi-fluid model on $400 \times 200 \times 10$ mesh.

\subsection{Importance of treatment of polydispersity}

Our objective in this section is to highlight the key role of polydispersity in the description of the dynamics of the droplets. We consider the same free-jet configuration as detailed previously but with a constant size distribution of the injected spray. We compare results obtained using one and ten size sections for the evaporative case. The constant mass-transfer number is set as $\mathrm{Bm}=0.1$. The corresponding non-dimensional evaporation coefficient is $K=0.07$. The Stokes number of the droplets in the one-section case is St $=1.88\left(d_{0}=\right.$ $119 \mu \mathrm{m})$. In the case of ten sections, the Stokes number ranges from $\mathrm{St}=0.0188\left(d_{0}=\right.$ $12 \mu \mathrm{m})$ to $2.86\left(d_{0}=147 \mu \mathrm{m}\right)$. Two results are provided, the first shows the spray number density, and the second the gas-phase fuel mass fraction.

When focusing on the number density (Figure 5), it is obvious that the global evaporation rate strongly depends on the refinement of the description of polydispersity. The evaporation, when considering one section, is highly underestimated in comparison to the evaporation when considering ten sections. This can be understood by considering the trans- 

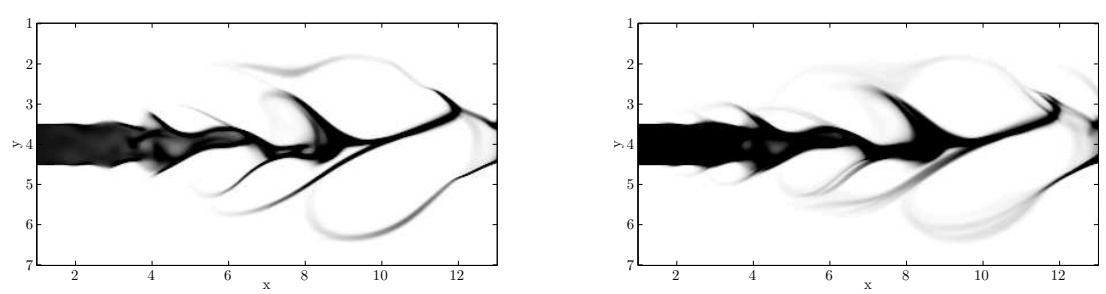

Fig. 5 Total number density of the polydisperse evaporating spray at time $t=20$. (Left) Multi-fluid model with one section. (Right) Multi-fluid model with ten sections.
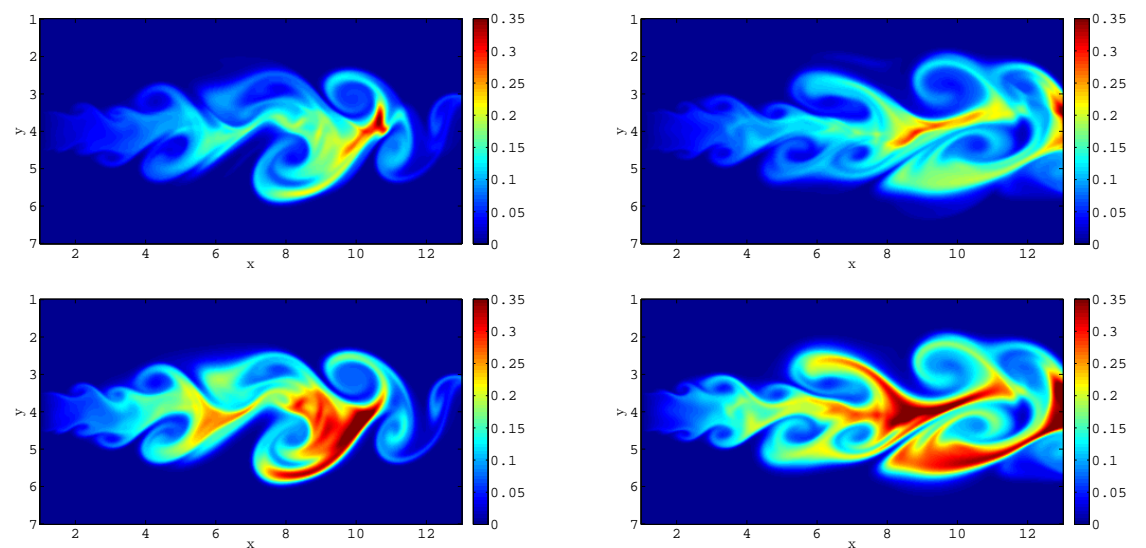

Fig. 6 Comparison of the gas-phase fuel mass fraction at times $t=15$ (left) and $t=20$ (right). (Top) Multifluid model with one section. (Bottom) Multi-fluid model with ten sections.

fer coefficients given in Eq. (4). For the higher sections, the evaporative coefficients $E_{2}^{(k)}$ are lower than the global coefficient in the case with one section. The opposite is true for the lower sections. Adding the fact that there is a mass flux from the higher sections to the lower ones leads us to the result of Figure 5. Backing up this conclusion, it can be seen in Figure 6 that the gas-phase fuel mass is higher in the computation with ten sections.

Furthermore, the dynamics observed are quite different for the spray with one section than for the spray with ten sections. First, as can be seen in Figure 6, when we focus on the free outlet zone, the gas-phase fuel mass fraction is higher with one section than with ten sections, whereas the opposite is true everywhere else in the domain. Indeed, the high evaporation rate has almost made the totality of the spray disappear, so that at the very end of the jet, only small droplets with low mass remain. On the contrary, with one section, the spray does not evaporate at as high a rate, which leads to the situation where the remaining liquid mass is much higher with one section than with ten sections. Thus the evaporation rate, proportional to the mass, becomes higher with one section.

A purely dynamic effect is observed in the gas-phase vortex interacting with the droplets whose repartition within the vortex depends on their size. For the one-section case, there is no segregation as a unique size is considered. In particular, there are no droplets at the center of the vortex. In contrast, with ten sections the segregation by size is significant. The bigger droplets are on the outer edge of the vortex, whereas the smaller ones remain near 
the center. These differences between the two models with respect to polydispersity have far-reaching consequences, since the accurate localization of gas-phase fuel mass fraction is a key requirement for combustion applications.

\subsection{Limitations of multi-fluid model}
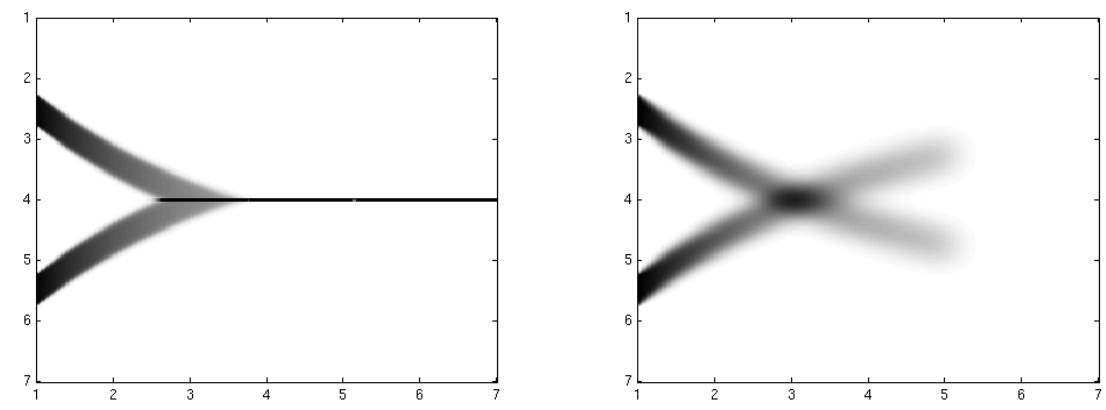

Fig. 7 Simulation of crossing jets with drag and evaporation at time time $t=10$. (Left) Multi-fluid model. (Right) Multi-fluid, multi-velocity model.

One typical configuration for which the multi-fluid model predicts an artificial spatial averaging is when two droplet jets cross for a monodisperse spray. Indeed, at the crossing point, there exist at the same space and time location two velocities leading to a bi-modal velocity distribution that is out of equilibrium. This configuration is presented in Figure 7. In Figure 7-right the multi-fluid, multi-velocity model, presented in the next section, can describe the crossing of the jets. Nevertheless, due to evaporation and drag, the CFL number is no longer unitary and some numerical diffusion appears. Because of the equilibrium assumption [H1], the multi-fluid method can not handle this case. Indeed, only different size droplets can experience crossing within the multi-fluid framework. If the multi-fluid model is used to describe dilute (non-collisional) flows, it results in the artificial collisional "zero-Knudsen" limit presented in Figure 7-left where a $\delta$-shock is created (i.e., mass accumulates on 1-D spatial structures). The presence of $\delta$-shocks is especially problematic for fully two-way coupled systems because mass accumulation at a $\delta$-shock can induce strong (unphysical) changes in the gas-phase fluid dynamics. For this reason, it is necessary to develop Eulerian models for non-equilibrium velocity distributions.

\section{Eulerian multi-fluid, multi-velocity approach}

\subsection{Multi-velocity approach for monodisperse sprays}

As shown in the previous section, dilute sprays with finite Stokes number particles can lead to particle trajectory crossings, which cannot be captured by multi-fluid models. In order to overcome this limitation, it is necessary to have recourse to a model that can capture 
multiple particle velocities at the same time and spatial location. In quadrature-based moment methods, the velocity distribution function is represented by a finite sum of weighted delta functions centered at discrete velocities [8-10]. These velocities, as well as the weights multiplying the delta functions, evolve in space and time to reproduce a finite set of lowerorder velocity moments. Most importantly, this multi-velocity approach provides a realizable kinetic-based closure for the spatial fluxes of the moments [21]. For non-collisional systems (i.e., infinite Knudsen number), the multi-velocity approach allows for an exact description of particle trajectory crossing [7]. In this section, we describe the implementation of the multi-velocity approach to solving the Williams-Boltzmann equation for a monodisperse spray in two dimensions corresponding to Eq. (1) with $K=0$ and $S=1$.

\subsubsection{Moment transport equations}

To handle the velocity moments, we employ a third-order moment closure using quadrature [8]. In two dimensions, the set of ten velocity moments up to third order is defined by

$$
W^{2}=\left(M_{00}, M_{10}, M_{01}, M_{20}, M_{11}, M_{02}, M_{30}, M_{21}, M_{12}, M_{03}\right) .
$$

The velocity moments are found from the velocity distribution function for a monodisperse spray by integration:

$$
M_{i j}=\int u_{1}^{i} u_{2}^{j} f \mathrm{~d} \mathbf{u}
$$

The unclosed transport equations for the velocity moments can be easily found starting from Eq. (1):

$$
\begin{aligned}
\partial_{t} M_{00}+\partial_{x_{1}} M_{10}+\partial_{x_{2}} M_{01}=0 \\
\partial_{t} M_{10}+\partial_{x_{1}} M_{20}+\partial_{x_{2}} M_{11}=\frac{1}{\mathrm{St}}\left(U_{\mathrm{g} 1} M_{00}-M_{10}\right), \\
\partial_{t} M_{01}+\partial_{x_{1}} M_{11}+\partial_{x_{2}} M_{02}=\frac{1}{\mathrm{St}}\left(U_{\mathrm{g} 2} M_{00}-M_{01}\right), \\
\partial_{t} M_{20}+\partial_{x_{1}} M_{30}+\partial_{x_{2}} M_{21}=\frac{2}{\mathrm{St}}\left(U_{\mathrm{g} 1} M_{10}-M_{20}\right), \\
\partial_{t} M_{11}+\partial_{x_{1}} M_{21}+\partial_{x_{2}} M_{12}=\frac{1}{\mathrm{St}}\left(U_{\mathrm{g} 1} M_{01}+U_{\mathrm{g} 2} M_{10}-2 M_{11}\right), \\
\partial_{t} M_{02}+\partial_{x_{1}} M_{12}+\partial_{x_{2}} M_{03}=\frac{2}{\mathrm{St}}\left(U_{\mathrm{g} 2} M_{01}-M_{02}\right), \\
\partial_{t} M_{30}+\partial_{x_{1}} M_{40}+\partial_{x_{2}} M_{31}=\frac{3}{\mathrm{St}}\left(U_{\mathrm{g} 1} M_{20}-M_{30}\right), \\
\partial_{t} M_{21}+\partial_{x_{1}} M_{31}+\partial_{x_{2}} M_{22}=\frac{1}{\mathrm{St}}\left(2 U_{\mathrm{g} 1} M_{11}+U_{\mathrm{g} 2} M_{20}-3 M_{21}\right), \\
\partial_{t} M_{12}+\partial_{x_{1}} M_{22}+\partial_{x_{2}} M_{13}=\frac{1}{\mathrm{St}}\left(U_{\mathrm{g} 1} M_{02}+2 U_{\mathrm{g} 2} M_{11}-3 M_{12}\right), \\
\partial_{t} M_{03}+\partial_{x_{1}} M_{13}+\partial_{x_{2}} M_{04}=\frac{3}{\mathrm{St}}\left(U_{\mathrm{g} 2} M_{02}-M_{03}\right),
\end{aligned}
$$

where the terms on the right-hand sides are due to drag. The unclosed fourth-order terms in the moment transport equations $\left(M_{40}, \ldots, M_{04}\right)$ are closed using quadrature as described below. Note that because St is constant, the drag terms are closed and linear functions of the moments. The corresponding coefficient matrix is lower diagonal with eigenvalues equal to $-(i+j)$ for $M_{i j}$. In the absence of transport (i.e. using Strang splitting), the drag terms can be solved analytically. 


\subsubsection{Relationship between moments and quadrature nodes}

Quadrature-based moment methods distinguish themselves from other moment methods by the use of quadrature weights and abscissas to model the unclosed terms in the moment transport equations. Thus, when developing a quadrature method, an important task is to define the algorithm for computing the weights and abscissas from the moments [8,9]. Here we limit ourselves to quadrature formulas for moments up to third order, and use onedimensional product formulas [8]. Thus, the number of quadrature nodes in each direction of velocity phase space will be two.

Let $V_{4}=\left[\left(n_{\alpha}, \mathbf{U}_{\alpha}\right)\right]$ with $\alpha \in(1,2,3,4)$ denote the set of weights and abscissas for the 4-node quadrature approximation of $f$. Note that the set of quadrature nodes $V_{4}$ contains 12 unknowns (i.e. four weights, and four 2-component velocity vectors). To find the components of $V_{4}$, we work with the velocity moments up to third order, which are related to the quadrature weights and abscissas by

$$
\begin{gathered}
M_{00}=\sum_{\alpha=1}^{4} n_{\alpha}, M_{10}=\sum_{\alpha=1}^{4} n_{\alpha} U_{1 \alpha}, M_{01}=\sum_{\alpha=1}^{4} n_{\alpha} U_{2 \alpha} \\
M_{20}=\sum_{\alpha=1}^{4} n_{\alpha} U_{1 \alpha}^{2}, M_{11}=\sum_{\alpha=1}^{4} n_{\alpha} U_{1 \alpha} U_{2 \alpha}, M_{02}=\sum_{\alpha=1}^{4} n_{\alpha} U_{2 \alpha}^{2} \\
M_{30}=\sum_{\alpha=1}^{4} n_{\alpha} U_{1 \alpha}^{3}, M_{21}=\sum_{\alpha=1}^{4} n_{\alpha} U_{1 \alpha}^{2} U_{2 \alpha}, M_{12}=\sum_{\alpha=1}^{4} n_{\alpha} U_{1 \alpha} U_{2 \alpha}^{2}, M_{03}=\sum_{\alpha=1}^{4} n_{\alpha} U_{2 \alpha}^{3} .
\end{gathered}
$$

Below we describe an algorithm for finding $V_{4}$ from $W^{2}$ [8]. The inverse operation (finding $W^{2}$ from $V_{4}$ ) is Eq. (7), which we will refer to as projection. In general, it will not be possible to represent all possible moment sets in $W^{2}$ using weights and abscissas in $V_{4}$. We will therefore define the set of representable moments as $W^{2 \dagger} \subset W^{2}$.

\subsubsection{Quadrature-based closure of spatial fluxes}

The moment transport equations given above contain unclosed spatial flux terms. Using quadrature, these fluxes can be expressed in terms of the weights and abscissas:

$$
\begin{gathered}
M_{40}=\sum_{\alpha=1}^{4} n_{\alpha} U_{1 \alpha}^{4}, M_{31}=\sum_{\alpha=1}^{4} n_{\alpha} U_{1 \alpha}^{3} U_{2 \alpha}^{1}, M_{22}=\sum_{\alpha=1}^{4} n_{\alpha} U_{1 \alpha}^{2} U_{2 \alpha}^{2}, \\
M_{13}=\sum_{\alpha=1}^{4} n_{\alpha} U_{1 \alpha}^{1} U_{2 \alpha}^{3}, M_{04}=\sum_{\alpha=1}^{4} n_{\alpha} U_{2 \alpha}^{4} .
\end{gathered}
$$

Quadrature is also used to write the other spatial fluxes in terms of the weights and abscissas $[6,7]$. The fluxes are based on the kinetic description using a delta-function representation of the velocity distribution function:

$$
f(\mathbf{u})=\sum_{\alpha=1}^{4} n_{\alpha} \delta\left(\mathbf{u}-\mathbf{U}_{\alpha}\right) .
$$

For example, the negative and positive contributions to the flux terms in the $x_{1}$ direction for the zero-order moment are expressed as

$$
M_{10}^{-}=\sum_{\alpha=1}^{4} n_{\alpha} \min \left(0, U_{1 \alpha}\right) \quad \text { and } \quad M_{10}^{+}=\sum_{\alpha=1}^{4} n_{\alpha} \max \left(0, U_{1 \alpha}\right) .
$$


Likewise, the fluxes for higher-order moments have analogous forms [6-9].

We should note that the fluxes as defined above are not guaranteed to produce moments that can be represented by the proposed quadrature algorithm [8]. For this reason, after advancing the moments due to the spatial fluxes (or any other process that does not remain in $W^{2}$ ), it is necessary to project the moments back into $W^{2 \dagger}$. This is accomplished simply by using the moments to compute the weights and abscissas, and then using Eq. (7) to recompute the moments.

\subsubsection{Four-node quadrature}

Using the set of ten moments up to third order $W^{2}$, we seek to define a four-node quadrature. We begin by defining the mean particle velocity vector [8]:

$$
\mathbf{U}_{\mathrm{p}}=\left[\begin{array}{l}
M_{10} / M_{00} \\
M_{01} / M_{00}
\end{array}\right]
$$

and the velocity covariance matrix:

$$
\sigma=\left[\sigma_{i j}\right]=\left[\begin{array}{cc}
M_{20} / M_{00}-U_{\mathrm{p} 1}^{2} & M_{11} / M_{00}-U_{\mathrm{p} 1} U_{\mathrm{p} 2} \\
M_{11} / M_{00}-U_{\mathrm{p} 1} U_{\mathrm{p} 2} & M_{02} / M_{00}-U_{\mathrm{p} 2}^{2}
\end{array}\right] .
$$

The next step is to introduce a linear transformation $\mathbf{A}$ to diagonalize $\sigma$. The choice of the linear transformation is not unique, but we choose to use a variation of the Cholesky decomposition as described in Sect. 5.1.5 below. With this choice we introduce a twocomponent vector $\mathbf{X}=\left[X_{1} X_{2}\right]^{\mathrm{T}}$ defined by

$$
\mathbf{X}=\mathbf{A}^{-1}\left(\mathbf{u}-\mathbf{U}_{\mathrm{p}}\right) \quad \text { so that } \quad \mathbf{u}=\mathbf{A} \mathbf{X}+\mathbf{U}_{\mathrm{p}} .
$$

If we denote the first four moments of $X_{i}$ by $m_{i}^{k}, k \in(0,1,2,3)$, then they are related to the velocity moments by

$$
\begin{gathered}
m_{i}^{0}=1, m_{i}^{1}=0, m_{i}^{2}=1, \\
m_{i}^{3}=h_{i}\left(\mathbf{A}, \mathbf{U}_{\mathrm{p}}, M_{30} / M_{00}, \ldots, M_{03} / M_{00}\right),
\end{gathered}
$$

where $h_{i}$ depends, in general, on all ten third-order velocity moments [8].

Using the two-node quadrature formulas [8], the moments of $X_{i}$ can be inverted for $i \in(1,2)$ to find $\left(n_{(i) 1}, n_{(i) 2}, X_{(i) 1}, X_{(i) 2}\right)$ :

$$
\begin{gathered}
n_{(i) 1}=0.5+\gamma_{i}, X_{(i) 1}=-\left(\frac{1-2 \gamma_{i}}{1+2 \gamma_{i}}\right)^{1 / 2}, \\
n_{(i) 2}=0.5-\gamma_{i}, X_{(i) 2}=\left(\frac{1+2 \gamma_{i}}{1-2 \gamma_{i}}\right)^{1 / 2},
\end{gathered}
$$

where $\left(-1 / 2<\gamma_{i}<1 / 2\right)$

$$
\gamma_{i}=\frac{m_{i}^{3} / 2}{\left[\left(m_{i}^{3}\right)^{2}+4\right]^{1 / 2}} .
$$

The four-node quadrature approximation is then defined using the tensor product of the one-dimensional abscissas as

$$
V_{4}^{*}=\left[\left(n_{1}^{*}, X_{(1) 1}, X_{(2) 1}\right),\left(n_{2}^{*}, X_{(1) 1}, X_{(2) 2}\right),\left(n_{3}^{*}, X_{(1) 2}, X_{(2) 1}\right),\left(n_{4}^{*}, X_{(1) 2}, X_{(2) 2}\right)\right]
$$


where the (as yet) unknown weights $n_{\alpha}^{*}$ must obey the linear equations [8]

$$
\begin{aligned}
& n_{1}^{*}-n_{4}^{*}=n_{(1) 1}-n_{(2) 2}, \\
& n_{2}^{*}+n_{4}^{*}=n_{(2) 2}, \\
& n_{3}^{*}+n_{4}^{*}=n_{(1) 2} .
\end{aligned}
$$

The right-hand sides of Eq. (19) are known, and have the property that $n_{(1) 1}+n_{(1) 2}=1$ and $n_{(2) 1}+n_{(2) 2}=1$.

The linear system in Eq. (19) has rank three. We must therefore add another linear equation to define the four weights. For this purpose, we will use the cross moment $m_{12}^{2}=$ $\left\langle X_{1} X_{2}\right\rangle=0$, the value of which follows from the definition of $\mathbf{A}$. In terms of the weights and abscissas in Eq. (18), we have

$$
X_{(1) 1} X_{(2) 1} n_{1}^{*}+X_{(1) 1} X_{(2) 2} n_{2}^{*}+X_{(1) 2} X_{(2) 1} n_{3}^{*}+X_{(1) 2} X_{(2) 2} n_{4}^{*}=0 .
$$

The resulting system can be inverted analytically to find

$$
\begin{aligned}
& n_{1}^{*}=n_{(1) 1} n_{(2) 1}=\left(0.5+\gamma_{1}\right)\left(0.5+\gamma_{2}\right) \\
& n_{2}^{*}=n_{(1) 1} n_{(2) 2}=\left(0.5+\gamma_{1}\right)\left(0.5-\gamma_{2}\right) \\
& n_{3}^{*}=n_{(1) 2} n_{(2) 1}=\left(0.5-\gamma_{1}\right)\left(0.5+\gamma_{2}\right) \\
& n_{4}^{*}=n_{(1) 2} n_{(2) 2}=\left(0.5-\gamma_{1}\right)\left(0.5-\gamma_{2}\right) .
\end{aligned}
$$

Note that these weights are always non-negative.

In summary, the weights and abscissas in $V_{4}$ are found from those in $V_{4}^{*}$ using Eq. (14) to invert the abscissas and $n_{\alpha}=M^{0} n_{\alpha}^{*}$. The eight moments controlled in this process are

$$
W^{2 *}=\left(m^{0}, m_{1}^{1}, m_{2}^{1}, m_{1}^{2}, m_{12}^{2}, m_{2}^{2}, m_{1}^{3}, m_{2}^{3}\right) .
$$

Note that the two third-order moments in $W^{2 *}$ are a linear combination of the four thirdorder moments in $W^{2}$. Hence, $W^{2 *}$ is a subset of $W^{2}$ containing eight independent moments (instead of ten). However, given moments in $W^{2}$ it is straightforward to project them (using the weights and abscissas) into $W^{2 \dagger}$, i.e., the eight-dimensional moment subspace that can be represented by $V_{4}$ is $W^{2 \dagger}$. The overall procedure can be represented as [8]

$$
W^{2} \rightarrow W^{2 *} \leftrightarrow V_{4}^{*} \leftrightarrow V_{4} \leftrightarrow W^{2 \dagger} \subset W^{2},
$$

where a projection step is used to define $W^{2 \dagger}$.

\subsubsection{Choice of velocity covariance decomposition}

Here we describe the decomposition used in this work to define A. In two dimensions (or greater), the correspondence between the moment set and the set of quadrature weights and abscissas is not one-to-one. We transport the whole set of moments but effectively restrict the moment subspace recursively structured from the set of second-order velocity moments for which the correspondence is one-to-one, and insure that the velocity moment vector lives in this subspace. An additional difficulty is that the choice of the transformation matrix $\mathbf{A}$ is not unique. In this work, we use the Cholesky decomposition of the covariance matrix, defined such that $\mathbf{L}^{\mathrm{T}} \mathbf{L}=\sigma$. Indeed, there are fundamental grounds for using this decomposition rather than other methods. For example, defining the matrix $\mathbf{A}$ in terms of the eigenvectors of the covariance matrix is a good choice for the passive transport of a distribution function. 
However, because the velocity is a dynamic variable, a fundamental difficulty comes from the fact that the eigenvectors of $\sigma$ do not vary smoothly with its components. As a consequence, the fluxes computed from the abscissas are then discontinuous, leading to random fluctuations in the moments. In contrast, the Cholesky matrix $\mathbf{L}$ defines $\mathbf{A}$ in such a way that it varies smoothly with the components of $\sigma$ and, hence, the fluxes are well-behaved [8].

However, the Cholesky matrix is itself non-unique. If we introduce a rotation matrix, $\mathbf{R}$, the matrix $\mathbf{R L}$ is another candidate for the decomposition. This brings a disadvantage for the use of the Cholesky matrix: it depends on the ordering of the covariance matrix, and is thus different for each of the two permutations (six in three dimensions) of the coordinates corresponding to two $\mathbf{R}$ matrices (identity and rotation by $\pi / 2$ ). It is thus desirable to replace the two linear transformations $\mathbf{A}_{x}$ and $\mathbf{A}_{y}$ in the two preceding choices with a permutationinvariant linear transformation. Here we employ the half-angle between $\mathbf{A}_{x}$ and $\mathbf{A}_{y}$, which treats each direction in the same manner and is independent of the ordering of the covariance matrix. Moreover, this choice is stable and defines a subspace of the moment space in which the conserved variables live.

In the particular cases where the dispersion of the distribution function is null for at least one direction (the moment vector lies on the boundary of moment space), the Cholesky matrix $\mathbf{L}$ becomes singular. In order to be able to treat this case without introducing an artificial velocity variance in the system, we use, for this particular case, the eigenvectors of the covariance matrix, where only one of the two eigenvalues is non-zero. The quadrature in the direction where the velocity variance is null is trivial, but this does not prevent us from using the 1-D quadrature method in the other direction. Details of the resulting quadrature algorithm are given in Appendix 1.

\subsection{Multi-fluid, multi-velocity model for polydisperse sprays}

The quadrature-based method for velocity moments described in Sect. 5.1 has been integrated in the multi-fluid model, described in Sect. 3. The resulting model, which we call the multi-fluid, multi-velocity model, overcomes the limitations of the multi-fluid model by capturing the dynamics of the spray, even in the "infinite Knudsen limit", while describing polydispersity like the multi-fluid model does. The most notable advance compared to the multi-fluid model is that the multi-fluid, multi-velocity model allows droplet crossing in the configuration of two impinging jets.

In this section, we consider a polydisperse spray. The multi-fluid model presumes the form of the NDF $f(t, \mathbf{x}, S, \mathbf{u})=n(t, \mathbf{x}, S) \delta(\mathbf{u}-\overline{\mathbf{u}}(t, \mathbf{x}, S))$. The droplet phase space is then discretized into sections. The multi-fluid, multi-velocity model goes beyond the equilibrium hypothesis, so that in each section $k$ the NDF is written as: $f(t, \mathbf{x}, S, \mathbf{u})=n(t, \mathbf{x}, S) \phi^{(k)}(\mathbf{u}-$ $\overline{\mathbf{u}}(t, \mathbf{x}, S))$, where $\phi^{(k)}$ is the velocity distribution, a priori different from the Dirac distribution. In other words, it is a distribution function characteristic of section $k$, such that $\int_{\mathbf{u}} \phi^{(k)}(t, \mathbf{x}, \mathbf{u}) \mathrm{d} \mathbf{u}=1$. The size and velocity distributions are then independent in each section so that polydispersity and the size distributions are solved independently. In particular, we can use the quadrature-based expression for $\phi^{(k)}$ as a sum of weights and abscissas, capturing the lower-order moments of $\phi^{(k)}$ up to the third order.

Let, as in Sect. 5.1.2, $d=2$ denote the number of velocity phase-space dimensions. Moreover, let us work on a size section, delimited by the interval $\left[S_{k-1}, S_{k}\left[\right.\right.$. The mass $m^{(k)}$, and the mean velocity $\overline{\mathbf{u}}^{(k)}$ are no longer enough to reconstruct the NDF. We need, as in Sect. 5.1, a ten moment set (up to third-order velocity moments) corresponding to four sets 
of weights and abscissas. These moments are defined by

$$
m^{(k)} M_{i j}^{(k)}=\int_{S_{k-1}}^{S_{k}} \rho_{1} S^{3 / 2} \int_{\mathbf{u}} u_{1}^{i} u_{2}^{j} f \mathrm{~d} \mathbf{u} \mathrm{d} S
$$

with the convention $M_{00}^{(k)}=1$. The moments are tensorial products of the size moment $m^{(k)}$ and the velocity moments $M_{i j}^{(k)}$. Consequently, in each section, the velocity distribution "sees" a monodisperse distribution, and can be reconstructed using exactly the same quadrature method presented in Sect. 5.1. If the size and velocity moments were fully coupled, then the phase space would have three dimensions, and the quadrature method would be even more complex. (We recall that the moment-inversion algorithm is exact only for monovariate distributions. The fact that it works in a two-dimensional velocity phase space is already quite exceptional.)

We introduce now the system of equations for the multi-fluid, multi-velocity model:

$$
\begin{aligned}
\partial_{t} m^{(k)} M_{i j}^{(k)}+\partial_{x_{1}}\left(m^{(k)} M_{i+1 j}^{(k)}\right)+\partial_{x_{2}}\left(m^{(k)} M_{i j+1}^{(k)}\right) & = \\
& \left(E_{1}^{(k)}+E_{2}^{(k)}\right) m^{(k)} M_{i j}^{(k)}-E_{1}^{(k+1)} m^{(k+1)} M_{i j}^{(k+1)}+m^{(k)} \overline{\mathbf{F}}^{(k)}
\end{aligned}
$$

where the average drag force $\overline{\mathbf{F}}^{(k)}$ is obtained, as for the multi-fluid model, using the mean surface $S_{\text {mean }}^{(k)}$. The dynamics of the velocity moments within each size section are the same as explained in Sect. 5.1. For the evaporation operator, the mass and momentum fluxes in the multi-fluid model are replaced by the fluxes of all the moments. A remarkable consequence is that the velocity distribution in section $k$ can change from a monomodal to a bimodal distribution due to the fluxes from section $k+1$.

\subsection{Numerical methods}

As done for the multi-fluid model in Eq. (3), we use a Strang splitting algorithm to solve system (23), splitting the transport in physical space from the transport in phase space through evaporation and drag. For the transport in physical space, the system is still weakly hyperbolic and equivalent to a kinetic description, once a quadrature is designed. We also use a kinetic scheme [3] but first-order accurate in space and time [8] in order to strictly preserve the moment space during the reconstruction part of the algorithm, which guarantees that the eigenvalues of the covariance matrix are both non-negative. In our simulations, we aim at working also on the boundary of moment space since we want to tackle cases where the velocity distribution reduces to a monokinetic distribution and the proposed quadrature degenerates to the multi-fluid model when the covariance matrix is zero up to machine precision.

The preservation of the moment space is also important during transport in phase space. The local dynamical system corresponding to the phase transport in Eq. (23) can be rewritten $\mathrm{d}_{t} Y^{(k)}=\Phi\left(Y^{(k)}\right)$ with

$$
\begin{aligned}
Y^{(k)}= & \left(m^{(k)}, m^{(k)} M_{10}^{(k)}, m^{(k)} M_{01}^{(k)}, m^{(k)} M_{20}^{(k)}, m^{(k)} M_{11}^{(k)}, m^{(k)} M_{02}^{(k)},\right. \\
& \left.m^{(k)} M_{30}^{(k)}, m^{(k)} M_{21}^{(k)}, m^{(k)} M_{12}^{(k)}, m^{(k)} M_{03}^{(k)}\right) .
\end{aligned}
$$

This system is solved using an implicit Runge-Kutta Radau IIA method of order 5 with adaptive time steps. Whereas this resolution in the case of the multi-fluid model did not 
yield any difficulties, for the multi-velocity model it can lead to a non-realizable set of $Y^{(k)}$. The preservation of moment space is facilitated by working with the central moments:

$$
m^{(k)} \tilde{M}_{i j}^{(k)}=\int_{S_{k-1}}^{S_{k}} \rho_{1} S^{3 / 2} \int_{\mathbf{u}}\left(u_{1}-M_{10}^{(k)}\right)^{i}\left(u_{2}-M_{01}^{(k)}\right)^{j} f(t, \mathbf{x}, S, \mathbf{u}) \mathrm{d} \mathbf{u} \mathrm{d} S,
$$

for $i+j \geq 2$. The equations for the transport in phase space of the central moments are given in Appendix 2. Using these transport equations, even though they have additional nonlinear terms, the Radau solver can be adapted and yields a robust solver on the conservative central moments that strictly preserves the moment space and allows working up to the boundary of moment space (i.e., a monokinetic velocity distribution).

\section{Results for multi-fluid, multi-velocity model}

6.1 Multi-fluid, multi-velocity versus Lagrangian models for free-jet configuration

The configuration chosen for the simulation with the multi-fluid, multi-velocity model is the same free-jet configuration with gas-phase instabilities as described in Sect. 4.2. The unstationary gas-phase velocity field destabilizes the liquid phase, and because spatially separated droplet clouds will interact with different gas-phase vortices, the droplets may impinge at a later time. Nonetheless, the intensity of crossings is relatively low as only a small amount of liquid interacts with the vortices. Indeed, the range of eligible Stokes numbers for which droplet crossing can be observed is small. On the one hand, the Stokes number must be greater than a minimum value, $\mathrm{St}_{\mathrm{min}}$, above which droplets can be ejected from the vortices. On the other hand, the Stokes number must be lower than a maximum value, $\mathrm{St}_{\max }$, above which the liquid phase does not interact with the gas phase. In the freejet configuration, the range of Stokes numbers is $[0.48,1.1]$. Nevertheless, this configuration precisely highlights an important property of our model, which is the ability to capture simultaneously regions where the droplet 'temperature' (or velocity variance) ${ }^{3}$ is low, and areas where the droplet temperature is strictly equal to zero.

For the simulations with the multi-velocity model, the first step is to show a good level of agreement between the Eulerian and Lagrangian simulations for the non-evaporating test case. Figure 8-left presents a fair comparison between the droplet number density fields with a level of agreement similar to the level obtained in earlier figures. In order to quantify the ability of the method to capture droplet crossing, we have also plotted in Figure 8-(top right) one-half the trace of the velocity covariance matrix, which amounts to a droplet 'temperature' in the case of an isotropic velocity distribution. However, the droplet temperature is defined for all types of velocity distributions, including isotropic and anisotropic ones, and therefore the crossings may be difficult to discern from the temperature field. ${ }^{4}$ In order to characterize regions of anisotropy, and thus regions where droplet crossings might be more easily observed, we have also plotted the absolute value of the difference of the two eigenvalues of the velocity covariance matrix in Figure 8-(bottom right). This figure very beautifully complements the plot in Figure 8-(top right), indicating that droplet crossings occur throughout the flow field.

\footnotetext{
3 The droplet temperature should not be confused with the temperature of the liquid.

4 Since droplet collisions are excluded from Eq. (1), a non-zero droplet temperature automatically implies the presence of droplet clouds with different velocities at the same location.
} 

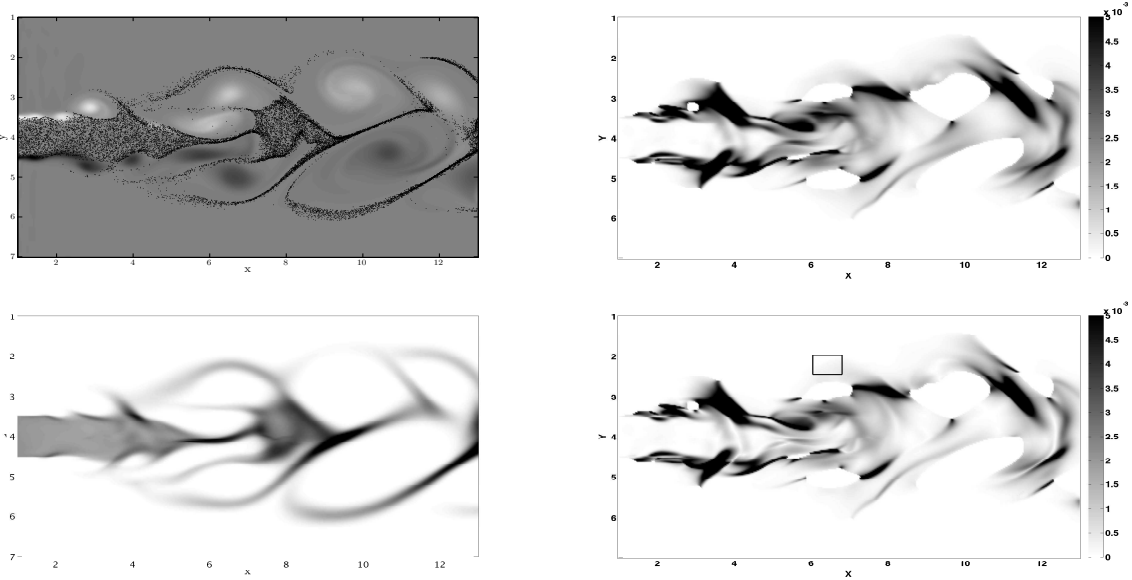

Fig. 8 Non-evaporating polydisperse spray with high-inertia droplets (Stokes 0.48 to 1.1 corresponding to diameters from 60 to $90 \mu \mathrm{m}$ ) at time $t=20$. (Top left) Lagrangian particle positions with 20,000 particles over gas vorticity. (Bottom left) Eulerian number density on $400 \times 200 \times 5$ grid. (Top right) Trace of velocity covariance matrix. (Bottom right) Absolute value of the difference between the two eigenvalues of the velocity covariance matrix.

Next, we focus a specific region of the flow domain in order to discuss details of the actual droplet velocity field. The region of interest is highlighted in Figure 8-(bottom right) and contains both a zone with large differences between the two eigenvalues of the velocity covariance matrix and a zone where the temperature is null. Figure 9-(top left) represents the velocity vectors in the first zone. The associated weights are displayed in Figure 9(top right) for the highest weights and in Figure 9-(bottom right) for the lowest weights and correspond, respectively, to the solid and bold arrows. As the order of magnitude between the two sets of weights is five, these figures show the ability of the multi-velocity model to capture the fine structure of the droplet jet. It can be easily seen that the two different types of velocity vectors correspond to two droplet clouds dragged by two different gas-phase vortices. Let us note that there can only be (except for very specific cases) two dominant velocity vectors, due to the fact that in the model we invert the velocity moment set using a two-node quadrature for each dimension. In the zero-temperature zone, it can be seen in Figure 9-(bottom left) that the velocity field consists of a single vector at each point. The important conclusion drawn from these figures is that the multi-velocity model (when carefully implemented) is able to capture both regions of droplet crossings as well as regions of zero temperature.

Finally, we have plotted the results of the multi-fluid, multi-velocity model with evaporation in the case of the polydisperse spray jet in Figure 10. Once again, this figure demonstrates the ability of the proposed method to capture the dynamics conditioned on size as well as evaporation for a range of small to moderate Stokes numbers.

6.2 Multi-velocity model versus multi-fluid model for crossing jets

In order to illustrate the behavior of the multi-velocity model in the context of a realistic jet, we use the same configuration as in Sect. 6, with the addition of a vertical jet of droplets that 

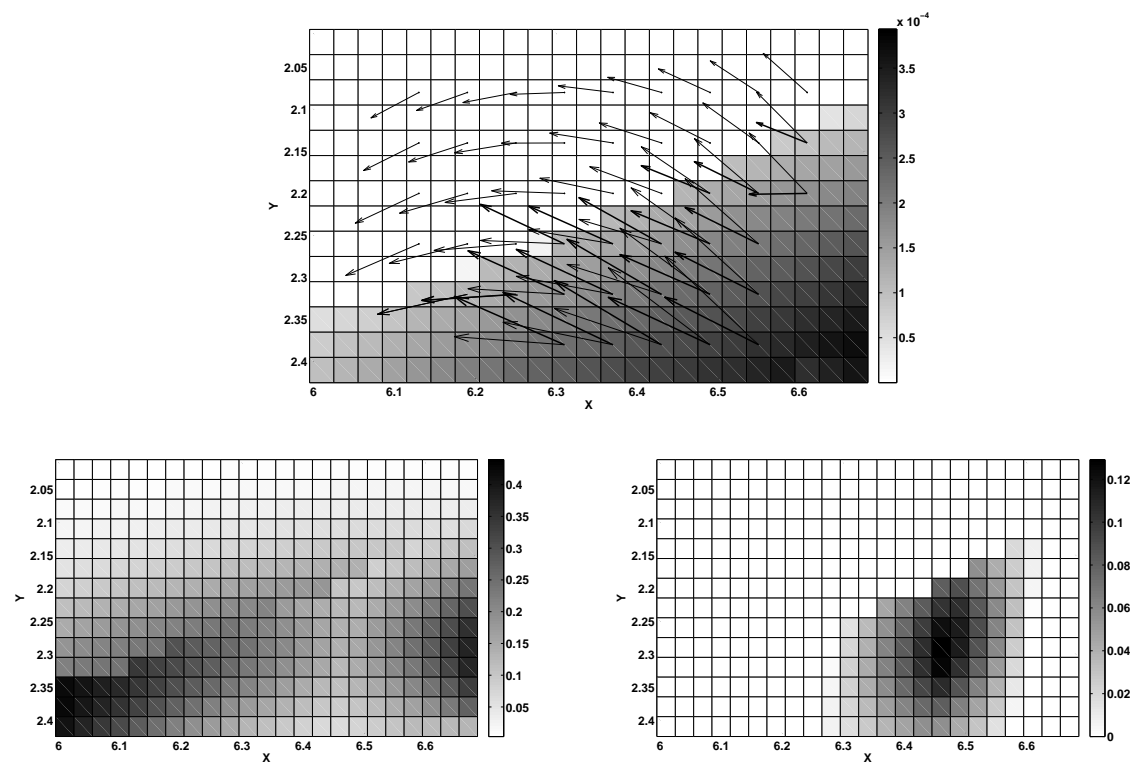

Fig. 9 Focus on region of the spray outlined by the rectangle in Fig. 8-(bottom right). (Top) Region where a significant and a null field of absolute value of the difference between the two eigenvalues of the velocity covariance matrix coexist. The two types of arrows (solid, bold) represent two different velocities and highlight droplet crossing in the zone where the absolute value of the difference between the two eigenvalues of the velocity covariance matrix is non-zero. In the zone where the droplet temperature is close to zero, the velocity field degenerates to one velocity. (Bottom left) Higher weights associated with the solid arrows. (Bottom right) Lower weights associated with the bold arrows.

will cross the horizontal jet. The gas phase is exactly the same as before and the droplets in the two jets are injected with the same velocity $\left(U_{0}\right)$, density and size. The particles in the vertical jet are inertial enough to cross the horizontal jet, even though they are decelerated by the gas. Their Stokes number is 4.05 , corresponding to a diameter of $175 \mu \mathrm{m}$. For comparison, the same configuration is simulated with a multi-fluid model with one section. In addition, separate simulations with only the horizontal or the vertical jet using the multi-velocity model are presented.

Results from the four simulations are given in Figure 11. The number density of the spray with two crossings jets obtained from the multi-velocity model is shown in Figure 11(top right). Results for the vertical jet are shown in Figure 11-(top left) and for the horizontal jet in Figure 11-(bottom left). One can see that the simulation of the two crossing jets corresponds to the superposition of the independent simulations of each jet. ${ }^{5}$ This behavior clearly illustrates the ability of the multi-velocity model to capture particle crossing. In contrast, the multi-fluid model in Figure 11-(bottom right) is unable to reproduce this kind of crossing (i.e. it cannot capture the exact solution to the Williams-Boltzmann equation) and instead produces a $\delta$-shock. As discussed in Sect. 4.4, the presence of $\delta$-shocks in a two-way coupled system will produce unphysical gas-phase flow structures.

\footnotetext{
5 In the absence of collisions, the Williams-Boltzmann equation is linear and thus the exact solution is a superposition.
} 

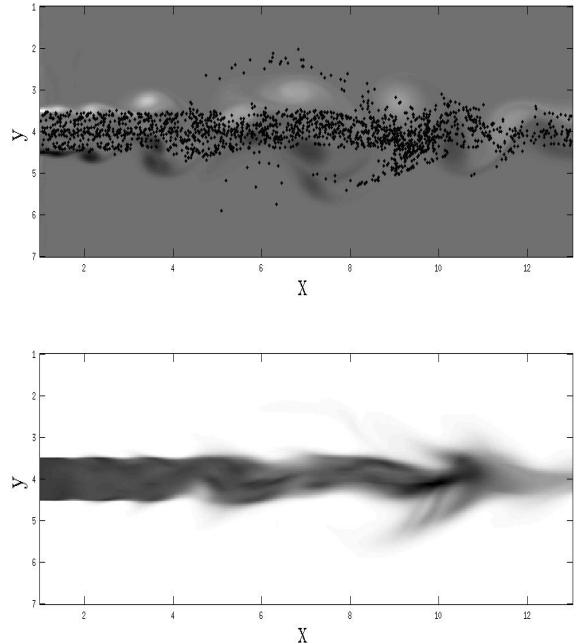

Fig. 10 Evaporating polydisperse spray with high-inertia droplets (Stokes 0.48 to 1.1 corresponding to diameters from 60 to $90 \mu \mathrm{m}$ ) at time $t=15$. (Top) Lagrangian particle positions with 7,000 particles over gas-phase vorticity. (Bottom) Eulerian number density on $400 \times 200 \times 10$ grid.
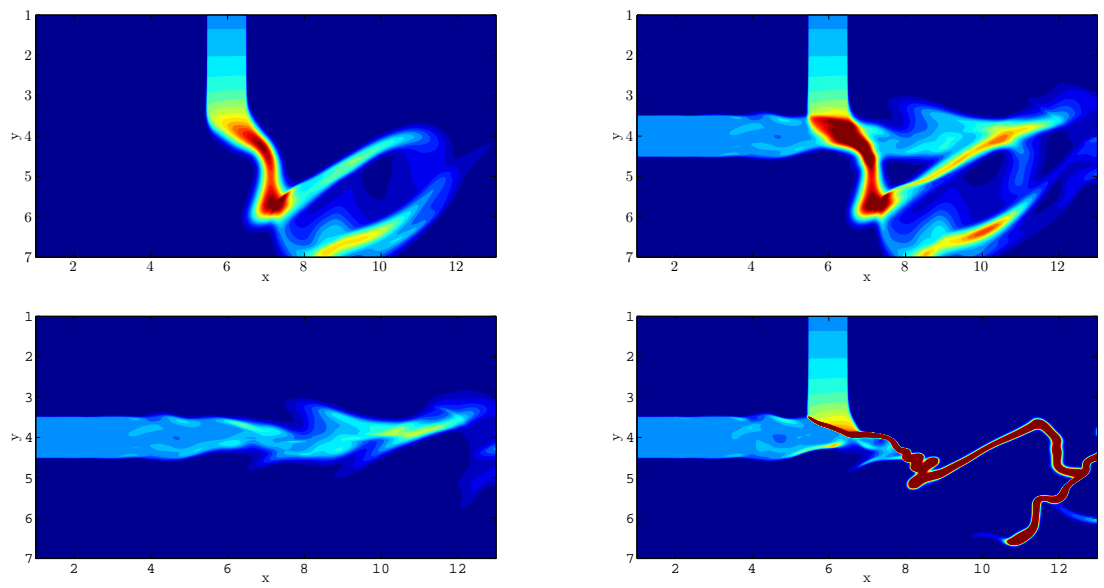

Fig. 11 Total number density of the non-evaporating spray at time $t=20$. (Top left) Vertical jet with the multi-velocity model. (Bottom left) Horizontal jet with the multi-velocity model. (Top right) Two crossing jets with the multi-velocity model. (Bottom right) Two crossing jets with the multi-fluid model.

\section{Conclusions}

Two types of Eulerian models for polydisperse evaporating sprays have been developed in this work. The first one, the multi-fluid model, has been demonstrated to give excellent agreement with Lagrangian simulations in a free-jet configuration with the injection of a polydisperse spray with and without evaporation. In addition, the gas-phase fuel mass 
fraction fields from the Eulerian model are in good agreement with the Lagrangian fields, while containing no statistical noise due to the finite number of numerical particles. By varying the number of sections in the multi-fluid model, we have shown the importance of including an accurate description of polydispersity when describing the gas-phase fuel mass fraction. Nevertheless, we demonstrated, using the example of crossing jets, that the multi-fluid model produces unphysical $\delta$-shocks. In order to overcome this limitation, we have developed a multi-velocity model that can accurately predict crossing jets in an Eulerian framework. By extending the multi-velocity model to include multiple sizes, the resulting multi-fluid, multi-velocity model can capture polydisperse sprays with droplets crossing in complex flow configurations, characteristic of spray combustion. In future work, the quadrature-based moment models will be extended to cases where the gas-phase velocity field is modeled by a large-eddy simulation.

Acknowledgements This research was supported by an ANR-05-JC05_42236 Young Investigator Award (M. Massot), by a DGA/CNRS Ph.D. grant for S. de Chaisemartin, and through a PEPS CNRS project (ST2I and MPPU - F. Laurent). Part of this work was performed during the 2008 Summer Program at the Center for Turbulence Research with financial support from Stanford University and NASA Ames Research Center.

\section{Appendix 1: Quadrature at boundary of moment space}

We distinguish between two different cases when the velocity covariance matrix becomes singular: (i) the singularity occurs in one of the two principal directions (i.e., $\sigma_{11}=0$ or $\sigma_{22}=0$ ), or (ii) it occurs in a non-principal direction. If the singularity occurs in a principal direction (let us choose $x_{1}$ as an example), then the diagonalisation is trivial. A onedimensional quadrature is performed on the moments in direction $x_{2}$ [8]. In direction $x_{1}$, one weight and the corresponding abscissa are set to one in order to conserve the droplet mass, the other weight and abscissa are null.

If the singularity does not occurs in a principal direction, the general relationship deduced from the fact that the velocity covariance matrix is singular is $\sigma_{12}^{2}=\sigma_{11} \sigma_{22}$ with $\sigma_{11} \neq 0$ and $\sigma_{22} \neq 0$. Letting $\rho=\sigma_{11} / \sigma_{12}$, the covariance matrix can be written as

$$
\sigma=\sigma_{12}\left[\begin{array}{cc}
\rho & 1 \\
1 & 1 / \rho
\end{array}\right]
$$

The eigenvalues of $\sigma$ are $\lambda_{1}=\sigma_{11}+\sigma_{22}$ and $\lambda_{2}=0$. The inverse transformation matrix for this case is

$$
\mathbf{A}^{-1}=\frac{1}{\alpha}\left[\begin{array}{cc}
\rho & 1 \\
-1 & \rho
\end{array}\right]
$$

with $\alpha=\left(\sigma_{11}-\sigma_{22}\right) / \sqrt{\sigma_{22}}$, given by the fact that $m_{1}^{2}=1$ in order to use Eq. (16) in the direction associated with eigenvalue $\lambda_{1}$. A one-dimensional quadrature is then performed on the moments in this direction [8]. In the orthogonal direction, like in the first case, one weight and the corresponding abscissa are set to one in order to conserve the droplet mass, the other weight and abscissa are null. The weights and abscissas in the canonical basis are defined using the relation $\mathbf{u}=\mathbf{A} \mathbf{X}+\mathbf{U}_{\mathrm{p}}$. 


\section{Appendix 2: Phase-space transport equations for central moments}

The central moments corresponding to moments of the distribution defined by Eq. (22) are

$$
\begin{aligned}
m^{(k)} \tilde{M}_{i j}^{(k)} & =\int_{S_{k-1}}^{S_{k}} \rho_{1} S^{3 / 2} \int_{\mathbf{u}}\left(u_{1}-M_{10}^{(k)}\right)^{i}\left(u_{2}-M_{01}^{(k)}\right)^{j} f(t, \mathbf{x}, S, \mathbf{u}) \mathrm{d} \mathbf{u} \mathrm{d} S \\
& =\sum_{p=0}^{i} \sum_{q=0}^{j}\left(\begin{array}{c}
i \\
p
\end{array}\right)\left(\begin{array}{c}
j \\
q
\end{array}\right)\left(-M_{10}^{(k)}\right)^{i-p}\left(-M_{01}^{(k)}\right)^{j-q} m^{(k)} M_{p q}^{(k)} .
\end{aligned}
$$

The part of Eqs. (23) corresponding to transport in the phase space through evaporation and drag can be rewritten in terms of the central moments:
$\mathrm{d}_{t}\left(m^{(k)} \tilde{M}_{00}^{(k)}\right)=-\left(E_{1}^{(k)}+E_{2}^{(k)}\right) m^{(k)}+E_{1}^{(k+1)} m^{(k+1)}$
$\mathrm{d}_{t}\left(m^{(k)} \tilde{M}_{10}^{(k)}\right)=-\left(E_{1}^{(k)}+E_{2}^{(k)}\right) m^{(k)} \tilde{M}_{10}^{(k)}+E_{1}^{(k+1)} m^{(k+1)} \tilde{M}_{10}^{(j+1)}+m^{(k)} \frac{U_{\mathrm{g} 1}-\tilde{M}_{10}^{(k)}}{\operatorname{St} S_{\text {mean }}^{(k)}}$
$\mathrm{d}_{t}\left(m^{(k)} \tilde{M}_{01}^{(k)}\right)=-\left(E_{1}^{(k)}+E_{2}^{(k)}\right) m^{(k)} \tilde{M}_{01}^{(k)}+E_{1}^{(k+1)} m^{(k+1)} \tilde{M}_{01}^{(j+1)}+m^{(k)} \frac{U_{\mathrm{g} 2}-\tilde{M}_{01}^{(k)}}{\operatorname{St} S_{\text {mean }}^{(k)}}$

$\mathrm{d}_{t}\left(m^{(k)} \tilde{M}_{20}^{(k)}\right)=-\left(E_{1}^{(k)}+E_{2}^{(k)}\right) m^{(k)} \tilde{M}_{20}^{(k)}+E_{1}^{(k+1)} m^{(k+1)} \tilde{M}_{20}^{(j+1)}-2 m^{(k)} \frac{\tilde{M}_{20}^{(k)}}{\operatorname{St} S_{\text {mean }}^{(k)}}$ $+E_{1}^{(k+1)} m^{(k+1)}\left(\tilde{M}_{10}^{(j+1)}-\tilde{M}_{10}^{(k)}\right)^{2}$

$\mathrm{d}_{t}\left(m^{(k)} \tilde{M}_{11}^{(k)}\right)=-\left(E_{1}^{(k)}+E_{2}^{(k)}\right) m^{(k)} \tilde{M}_{11}^{(k)}+E_{1}^{(k+1)} m^{(k+1)} \tilde{M}_{11}^{(j+1)}-2 m^{(k)} \frac{\tilde{M}_{11}^{(k)}}{\operatorname{St} S_{\text {mean }}^{(k)}}$ $+E_{1}^{(k+1)} m^{(k+1)}\left(\tilde{M}_{10}^{(j+1)}-\tilde{M}_{10}^{(k)}\right)\left(\tilde{M}_{01}^{(j+1)}-\tilde{M}_{01}^{(k)}\right)$

$\mathrm{d}_{t}\left(m^{(k)} \tilde{M}_{02}^{(k)}\right)=-\left(E_{1}^{(k)}+E_{2}^{(k)}\right) m^{(k)} \tilde{M}_{02}^{(k)}+E_{1}^{(k+1)} m^{(k+1)} \tilde{M}_{02}^{(j+1)}-2 m^{(k)} \frac{\tilde{M}_{02}^{(k)}}{\operatorname{St} S_{\text {mean }}^{(k)}}$ $+E_{1}^{(k+1)} m^{(k+1)}\left(\tilde{M}_{01}^{(j+1)}-\tilde{M}_{01}^{(k)}\right)^{2}$ 
$\mathrm{d}_{t}\left(m^{(k)} \tilde{M}_{30}^{(k)}\right)=-\left(E_{1}^{(k)}+E_{2}^{(k)}\right) m^{(k)} \tilde{M}_{30}^{(k)}+E_{1}^{(k+1)} m^{(k+1)} \tilde{M}_{30}^{(j+1)}-3 m^{(k)} \frac{\tilde{M}_{30}^{(k)}}{\operatorname{St} S_{\text {mean }}^{(k)}}$

$$
+E_{1}^{(k+1)} m^{(k+1)}\left[3\left(\tilde{M}_{20}^{(j+1)}-\tilde{M}_{20}^{(k)}\right)\left(\tilde{M}_{10}^{(j+1)}-\tilde{M}_{10}^{(k)}\right)+\left(\tilde{M}_{10}^{(j+1)}-\tilde{M}_{10}^{(k)}\right)^{3}\right]
$$

$\mathrm{d}_{t}\left(m^{(k)} \tilde{M}_{21}^{(k)}\right)=-\left(E_{1}^{(k)}+E_{2}^{(k)}\right) m^{(k)} \tilde{M}_{21}^{(k)}+E_{1}^{(k+1)} m^{(k+1)} \tilde{M}_{21}^{(j+1)}-3 m^{(k)} \frac{\tilde{M}_{21}^{(k)}}{\operatorname{St} S_{\text {mean }}^{(k)}}$

$$
\begin{aligned}
& +E_{1}^{(k+1)} m^{(k+1)}\left[\left(\tilde{M}_{20}^{(j+1)}-\tilde{M}_{20}^{(k)}\right)\left(\tilde{M}_{01}^{(j+1)}-\tilde{M}_{01}^{(k)}\right)\right. \\
& \left.+2\left(\tilde{M}_{11}^{(j+1)}-\tilde{M}_{11}^{(k)}\right)\left(\tilde{M}_{10}^{(j+1)}-\tilde{M}_{10}^{(k)}\right)+\left(\tilde{M}_{01}^{(j+1)}-\tilde{M}_{01}^{(k)}\right)\left(\tilde{M}_{10}^{(j+1)}-\tilde{M}_{10}^{(k)}\right)^{2}\right]
\end{aligned}
$$

$\mathrm{d}_{t}\left(m^{(k)} \tilde{M}_{12}^{(k)}\right)=-\left(E_{1}^{(k)}+E_{2}^{(k)}\right) m^{(k)} \tilde{M}_{12}^{(k)}+E_{1}^{(k+1)} m^{(k+1)} \tilde{M}_{12}^{(j+1)}-3 m^{(k)} \frac{\tilde{M}_{12}^{(k)}}{\operatorname{St} S_{\text {mean }}^{(k)}}$

$$
\begin{aligned}
& +E_{1}^{(k+1)} m^{(k+1)}\left[\left(\tilde{M}_{02}^{(j+1)}-\tilde{M}_{02}^{(k)}\right)\left(\tilde{M}_{10}^{(j+1)}-\tilde{M}_{10}^{(k)}\right)\right. \\
& \left.+2\left(\tilde{M}_{11}^{(j+1)}-\tilde{M}_{11}^{(k)}\right)\left(\tilde{M}_{01}^{(j+1)}-\tilde{M}_{01}^{(k)}\right)+\left(\tilde{M}_{01}^{(j+1)}-\tilde{M}_{01}^{(k)}\right)^{2}\left(\tilde{M}_{10}^{(j+1)}-\tilde{M}_{10}^{(k)}\right)\right]
\end{aligned}
$$

$\mathrm{d}_{t}\left(m^{(k)} \tilde{M}_{03}^{(k)}\right)=-\left(E_{1}^{(k)}+E_{2}^{(k)}\right) m^{(k)} \tilde{M}_{03}^{(k)}+E_{1}^{(k+1)} m^{(k+1)} \tilde{M}_{03}^{(j+1)}-3 m^{(k)} \frac{\tilde{M}_{03}^{(k)}}{\operatorname{St} S_{\text {mean }}^{(k)}}$

$$
+E_{1}^{(k+1)} m^{(k+1)}\left[3\left(\tilde{M}_{02}^{(j+1)}-\tilde{M}_{02}^{(k)}\right)\left(\tilde{M}_{01}^{(j+1)}-\tilde{M}_{01}^{(k)}\right)+\left(\tilde{M}_{01}^{(j+1)}-\tilde{M}_{01}^{(k)}\right)^{3}\right]
$$

\section{References}

1. Bardos, C., Golse, F., Levermore, D.: Fluid dynamic limits of kinetic equations. I. Formal derivations. J. Statist. Phys. 63(1-2), 323-344 (1991)

2. Bird, G.A.: Molecular gas dynamics and the direct simulation of gas flows, Oxford Engineering Science Series, vol. 42. Oxford University Press (1994)

3. Bouchut, F., Jin, S., Li, X.: Numerical approximations of pressureless and isothermal gas dynamics. SIAM J. Numer. Anal. 41(1), 135-158 (2003)

4. de Chaisemartin, S.: Modèles eulériens et simulation numérique de la dispersion turbulente de brouillards qui s'évaporent. Ph.D. thesis, Ecole Centrale Paris, France (2009). Available in English on TEL (http://tel.archives-ouvertes.fr/tel-00443982/en/)

5. de Chaisemartin, S., Laurent, F., Massot, M., Reveillon, J.: Evaluation of Eulerian multi-fluid versus Lagrangian methods for ejection of polydisperse evaporating sprays by vortices. European project report, TIMECOP-AE Project (2007). Available on HAL: http://hal.archives-ouvertes.fr/hal-00169721/en/

6. Desjardins, O., Fox, R.O., Villedieu, P.: A quadrature-based moment closure for the Williams spray equation. In: Proceedings of the Summer Program 2006, Center for Turbulence Research, Stanford University, pp. 223-234 (2006)

7. Desjardins, O., Fox, R.O., Villedieu, P.: A quadrature-based moment method for dilute fluid-particle flows. J. Comput. Phys. 227(12), 6313-6350 (2008)

8. Fox, R.O.: A quadrature-based third-order moment method for dilute gas-particle flow. J. Comput. Phys. 227(12), 6313-6350 (2008)

9. Fox, R.O.: Higher-order quadrature-based moment methods for kinetic equations. J. Comput. Phys. 228(20), 7771-7791 (2009)

10. Fox, R.O., Laurent, F., Massot, M.: Numerical simulation of spray coalescence in an Eulerian framework: direct quadrature method of moments and multi-fluid method. J. Comput. Phys. 227(6), 3058-3088 (2008)

11. Fox, R.O., Vedula, P.: Quadrature-based moment model for moderately dense polydisperse gas-particle flows. IEC Research (in press), 1-13 (2009) 
12. Freret, L., de Chaisemartin, S., Laurent, F., Vedula, P., Fox, R.O., Thomine, O., Reveillon, J., Massot, M.: Eulerian moment models for polydisperse weakly collisional sprays: model and validation. In: Proceedings of the Summer Program 2008, Center for Turbulence Research, Stanford University, pp. 277-288 (2009)

13. Grad, H.: Principles of the kinetic theory of gases. In: Handbuch der Physik (herausgegeben von S. Flügge), Bd. 12, Thermodynamik der Gase, pp. 205-294. Springer-Verlag, Berlin (1958)

14. Greenberg, J.B., Silverman, I., Tambour, Y.: On the origin of spray sectional conservation equations. Combustion and Flame 93, 90-96 (1993)

15. Klein, M., Sadiki, A., Janicka, J.: A digital filter based generation of inflow data for spatially developing direct numerical or large eddy simulations. J. Comput. Phys. 186, 652-665 (2003)

16. Laurent, F., Massot, M.: Multi-fluid modeling of laminar poly-dispersed spray flames: origin, assumptions and comparison of sectional and sampling methods. Comb. Theory and Modelling 5, 537-572 (2001)

17. Laurent, F., Santoro, V., Noskov, M., Gomez, A., Smooke, M., Massot, M.: Accurate treatment of size distribution effects in polydispersed spray diffusion flames: multi-fluid modeling, computations and experiments. Combust. Theory and Modelling 8, 385-412 (2004)

18. Massot, M.: Eulerian multi-fluid models for polydisperse evaporating sprays. In: Multiphase reacting flows: modelling and simulation, CISM Courses and Lectures, vol. 492, pp. 79-123. SpringerWienNewYork, Vienna (2007). Editors D.L. Marchisio and R. O. Fox, Udine, July 2006

19. Massot, M., Laurent, F., de Chaisemartin, S., Fréret, L., Kah, D.: Eulerian multi-fluid models: modeling and numerical methods. In: Modelling and Computation of Nanoparticles in Fluid Flows, Lectures of the von Karman Institue. NATO RTO (2009). In Press, available on HAL : http://hal.archives-ouvertes.fr/hal00423031/en/

20. Massot, M., Laurent, F., Kah, D., de Chaisemartin, S.: A robust moment method for evaluation of the disappearance rate of evaporating sprays. SIAM J. Appl. Math. (2010). Accepted for publication, http://hal.archives-ouvertes.fr/hal-00332423/en/

21. Perthame, B.: Second-order Boltzmann schemes for compressible Euler equations in one and two space dimensions. SIAM J. Numer. Anal. 29(1), 1-19 (1992). DOI 10.1137/0729001. URL http://dx.doi.org/10.1137/0729001

22. Pope, S.B.: PDF methods for turbulent reactive flows. Progress in Energy and Combustion Science 11, 119-192 (1985)

23. Reveillon, J.: DNS of spray combustion, dispersion evaporation and combustion. In: Computational Models for Turbulent Multiphase Reacting Flows, CISM Courses and Lectures, vol. 492, p. 229. SpringerWienNew York, Vienna (2007). Editors D.L. Marchisio and R. O. Fox, Udine, July 2006

24. Reveillon, J., Demoulin, F.X.: Effects of the preferential segregation of droplets on evaporation and turbulent mixing. J. Fluid Mech. 583, 273-302 (2007)

25. Reveillon, J., Péra, C., Massot, M., Knikker, R.: Eulerian analysis of the dispersion of evaporating polydispersed sprays in a statistically stationary turbulent flow. J. Turbulence 5(1), 1-27 (2004)

26. Williams, F.A.: Spray combustion and atomization. Phys. Fluids 1, 541-545 (1958) 